Mathematical Programming manuscript No.

(will be inserted by the editor)

José R. Rodríguez-Mancilla · William T. Ziemba

\title{
The Duality of Option Investment Strategies for Hedge Funds *
}

Received: July 2003 /

Abstract. This paper explores the structure of optimal investment strategies using stochastic programming and duality theory in investment portfolios containing options for a hedge fund manager who attempts to beat a benchmark. Explicit optimal conditions for option investments are obtained for several models.

\section{Introduction}

Hedge funds ${ }^{1}$ are private investment partnerships that attempt to obtain superior risk adjusted returns in any market condition for their mostly wealthy investors. Although hedge funds have long existed ${ }^{2}$, it was not until the collapse of the Long-Term Capital Management hedge fund (LTCM) in 1998 that it was fully realized the influence and role that these institutions play in the financial markets (see de Brouwer (2001). The high-profile (4 billion dollars) failure of LTCM jeopardized several large financial institutions and, according to the U.S. Federal Reserve, the world economy. The reasons for this near-catastrophic event were the highly leveraged position of LTCM and the poor risk management that underestimated the risk of such position when a bad scenario occurred (See Jorion (2001), de Brouwer (2001), and Ziemba (2003)). Therefore, appropriate risk management of hedge funds strategies is crucial.

Hedge funds have been studied in the recent academic literature. A major part of this literature has empirically studied hedge funds performance and risk. Some have described their organization or strategies (Fung et al (1997)) while others have attempted to estimate returns or to measure performance (Ackermann et al (1999) and Kouwenberg (2003)). The theoretical literature is small and has mainly focused on finding optimal fee structures (Heinkel and Stoughton (1994), Maug and Naik (1995), and Dybvig et al (2000)), exploiting arbitrage opportunities (Liu and Longstaff (2000) and Lowenstein and Willard (2000)), and computing the incentive-fees of hedge fund managers (Goetzmann et al (2003)). Besides the literature on the use of arbitrage opportunities strategies, that focus more in equilibrium

J.R. Rodríguez-Mancilla: Sauder School of Business at the University of British Columbia, Vancouver, Canada.

W.T. Ziemba: Sauder School of Business at the University of British Columbia, Vancouver, Canada and Swiss Banking Institute, University of Zurich, Zurich, Switzerland.

Mathematics Subject Classification (1991): 20E28, 20G40, 20C20

* This research was supported by Inquire. Additional support was obtained from the Social Sciences and Humanities Research Council of Canada, the Natural Sciences and Engineering Research Council of Canada and the National Center of Competence in Research FINRISK, a research instrument of the Swiss National Science Foundation.

1 The term hedge fund was coined to describe the "hedge" against risk that some of these partnerships aim for through their strategies.

2 The first official hedge fund was founded in 1949 by Alfred Winslow Jones in the United States. For more details on the history of hedge funds see Eichengreen et al (1998). Early private hedge funds, such as the Chest Fund at King's College (Cambridge) which was managed by the famous economist J.M. Keynes from 1927 - 1945, are discussed in Ziemba (2003). 
models rather than arbitrage strategies, there are some papers that study or propose optimal strategies for a hedge fund manager (see Ziemba(2003)). For instance, Carpenter (2000) solves for the optimal dynamic portfolio for a convex compensation of a manager (such as a hedge fund manager). Kouwenberg and Ziemba (2003), using a prospect theory utility function, investigate how manager's incentive fees affect the hedge fund manager's risk taking. They find that incentives increase risk but that risk is greatly reduced if the manager has a substantial amount of own money in the fund. In an empirical study they find that hedge funds with incentive fees have higher downside risk than funds without such compensation contracts and that mean returns in absolute and risk adjusted terms are significantly lower in the presence of incentive fees. The convex compensation problem applies to a variety problems other than a hedge fund manager. For instance, a corporate manager that controls firm leverage or the compensation of a trader at a securities firm. However, Carpenter's paper and most of the theoretical literature develop their models in a continuous-time framework under the assumption that the security prices follow Brownian motion processes and hence under the assumption that the security returns are normally distributed, which has the serious drawback of not modelling the fat tails of real asset prices distributions that are commonly observed for high-frequency sampled returns (e.g. daily) for both stock indexes and individual stocks (See Campbell et al (1997)) and in portfolios containing options. One of the principal reasons for the Brownian motion assumption is the vast mathematical theory that has been developed about it (e.g. Karatzas and Shreve (1991)) and that allows in many cases to obtain explicit expressions (e.g. Carpenter (2000)). Other drawback is the usual assumption of complete markets (e.g. Duffie (2001)) which often does not hold in reality.

This paper studies the structure of optimal investment strategies for a hedge fund manager, who is typically the general partner and thus a major investor in the fund, using stock index options in the hedge fund's portfolio in a discrete-time framework that overcomes some of the drawbacks of the continuous-time framework. It is able to model fat tails and does not require market completeness. This paper also studies models that address risk management of underperformance with respect to the benchmark, option investments, as well as liquidity and short selling restrictions.

This paper provides explicit optimal conditions for stock-index-option investments in terms of embedded martingale measures using duality theory. Explicit relationships between these martingale measures and the hedge fund manager preferences are derived.

Sections two and three describe, respectively, the hedge fund manager's problem and the decision space framework. Sections four and five analyze optimal strategies when there exists a single period (the terminal period) in which the hedge fund manager receives a fee. Section four provides the basic assumptions about the dynamics of the portfolio's value used in the paper, and studies optimal strategies when the goal is to maximize the hedge fund manager's expected net fee. Section five studies the case in which the hedge fund manager maximizes only the expected utility of his fee. Section six treats the case of multiple periods of fee payments. Section seven considers more advanced models, and section eight concludes.

\section{The Hedge Fund Manager's Problem}

The hedge fund manager controls assets with an initial value $W_{0}$ over a time horizon of $T$ periods. There exists monitoring periods $\left(t_{1}<\ldots<t_{p}, p \leq T\right)$ in which the manager receives a fee composed by a flat fee $f$ and a variable fee consisting of a percentage $\alpha$ on the positive profit over a sequence of random dependent benchmarks $\left\{B_{t_{i}}\right\}_{i=1, \ldots, p}$. The variety of incentive fees relies on the way this sequence of benchmarks is determined. Typical (Eichengreen et al (1998) and de Brower (2001)) policies include: 
- Fixed benchmarks, e.g. a fixed return within consecutive monitoring periods.

- Stock Index Benchmarks, e.g. S\&P500.

- "High water marks", which consists in setting the benchmark at time at time $t_{i}$ as the maximum net asset value, without discounting, since the inception of the fund, namely $B_{t_{i}}=\operatorname{Max}\left\{W_{t_{i-1}}, B_{t_{i-1}}\right\}$ for $i \geq 2$ and $B_{0}=B_{t_{1}}=W_{0}$.

\section{Decision Space Framework}

Our framework is based on King (2002) using three elements: a probability space, a certain type of financial market, and a particular class of investors.

\subsection{Probability Space}

The probability space uses a scenario tree structure that models all possible scenarios or states (represented by nodes of a tree) of the market over a finite number of discrete time periods $t=0, \ldots, T$. The scenario tree structure is such that every possible state is the consequence of a unique sequence (trajectory) of states (events). This is convenient, for assigning probabilities to each of the tree scenario nodes.

Assume that every node $n \epsilon N_{t}$ ( $N_{t}$ denotes the set of all nodes at time $t$ ) has a unique parent denoted by $a(n) \in N_{t-1}$ and a set of child nodes $C(n) \subseteq N_{t+1}$. Defining a probability measure (i.e. assigning probabilities to each node of the tree), say $P$, consists of assigning weights $p_{n}>0$ to each leaf node $n \epsilon N_{T}$ such that $\sum_{n \epsilon N_{T}} p_{n}=1$ and then recursively computing the remaining node probabilities

$$
p_{n}=\sum_{m \in C(n)} p_{m}, \forall n \epsilon N_{t}, t=T-1, \ldots, 0 .
$$

Let $\Omega$ be the set of possible trajectories or sequence of events (from time 0 to the end of period $T)$ in the scenario tree, then $(\Omega, P)$ defines a sample space. Every node $n \epsilon N_{t}$ has a unique history up to time $t$ and a unique set of possible future trajectories. $N_{t}$ induces a unique set of histories up to time $t$, say $F_{t}$, and a partition of $\Omega$. The collection of sets $\left\{F_{t}\right\}_{t=0, \ldots, T}$ satisfy $F_{t} \subseteq F_{t+1}$ for $t=0, \ldots, T-1$. The triplet $\left(\Omega, F_{T}, P\right)$ form a probability space. Hence, for a probability space $\left(\Omega, F_{T}, P\right)$, the conditional probability of state (event) $m$ given that $n$ occurs $(m \in C(n))$ is $\left(\frac{p_{m}}{p_{n}}\right)$, and if $\left\{X_{t}\right\}_{t=0, \ldots, T}$ is a discrete stochastic process (defined on our probability space), then $E^{P}\left[X_{t}\right]=\sum_{n \epsilon N_{t}} X_{n} p_{n}$ and $E^{P}\left[X_{t+1} \mid N_{t}\right]=\sum_{m \epsilon C(n)}\left(\frac{p_{m}}{p_{n}}\right) X_{m}$.

\section{Martingales}

Definition 1 If there exists a probability measure $Q$ such that

$$
Z_{t}=E^{Q}\left[Z_{t+1} \mid N_{t}\right], t=0, \ldots, T-1
$$

the process $\left\{Z_{t}\right\}_{t=0, \ldots, T}$ is called a martingale under $Q$ and $Q$ is called a martingale measure for the process $\left\{Z_{t}\right\}$.

Martingales are useful to determine if a certain price process is fair in the sense that at any time the price equates its expected future value (under a probability measure $Q$ ), i.e if the price process is a martingale (under $Q$ ). 


\subsection{Financial Market}

The market consists of $N+1$ tradable securities $i=0, \ldots, N$ and whose prices at node $n$ are $S_{n}=\left(S_{n}^{0}, \ldots, S_{n}^{N}\right)$. Assume that one of the securities, the numeraire, has always a strictly positive value and without loss of generality assume it is security 0. Define discount factors $\beta_{n}=\left(\frac{1}{S_{n}^{0}}\right) \forall n \in N_{t}$ and the discounted price (relative to the numeraire) $Z_{n}^{i} \equiv \beta_{n} S_{n}^{i}, \forall i=0, \ldots, N$ where $Z_{n}^{0}=1 \forall n \epsilon N_{t}, t=0, \ldots, T$. The market could be complete or incomplete.

3.2.1. Arbitrage Arbitrage refers to the opportunity of making a sure profit out of nothing (usually through the purchase and sale of assets). In our framework, arbitrage reduces to finding a portfolio with zero initial value whose terminal values, obtained through selffinancing strategies, are nonnegative for any scenario and for which at least one of those is strictly positive and has a positive probability of occurring. There is arbitrage if it exists $\left\{\theta_{n}\right\}_{n \in N_{t}, 0 \leq t \leq T}$ such that

$$
\begin{aligned}
& Z_{0} \cdot \theta_{0}=0 \\
& Z_{n} \cdot\left(\theta_{n}-\theta_{a(n)}\right)=0 \\
& Z_{n} \cdot \theta_{n} \geq 0 \quad \forall n \in N_{T}, \text { and } \\
& P\left\{Z_{m} \cdot \theta_{m}>0\right\}>0 \text { for some } m \in N_{T}
\end{aligned}
$$

We assume that there is no arbitrage in our financial market. This assumption guarantees, under this framework, the existence of martingale measures for $\left\{Z_{t}\right\}_{t=0, \ldots, T}$ (King (2002), Theorem 2.2) which are used to express optimal conditions for option investments for several models in subsequent sections.

\subsection{Investors}

We assume a class of investors that do not influence the prices of any security and who may trade at every time-step base on historical information up to time $t$. The amount of security $i$ held by the investor in state $n \epsilon N_{t}$ is $\theta_{n}^{i}$ and the portfolio value in state $n \epsilon N_{t}$ is

$$
Z_{n} \cdot \theta_{n} \equiv \sum_{i=0}^{N} Z_{n}^{i} \cdot \theta_{n}^{i}
$$

\section{Two Simple Strategies}

The two simple strategies that we consider in this section consist of investing the initial hedge fund capital in a portfolio of $N+1$ securities and to buy or sell options on a Stock Index, the usual benchmark, which deliver cash rather than stock by an amount equals the difference between the current level of the Stock Index and the exercise price times a certain factor N (e.g. 100 for SPX). 


\subsection{Basic Assumptions}

We begin by simplifying the hedge fund manager problem. Assume that the manager decides on the optimal investment strategy over a $T$-period horizon in which he attempts to beat a random benchmark (e.g. a stock index such as the S\&P500) or a fixed benchmark at the end of the such horizon. Our strategies are self-financing, given the common hedge fund policy of penalizing any fund withdrawn within consecutive monitoring periods (we have just one monitoring period), and the terminal value of the portfolio to be nonnegative in any scenario and hereafter forcing our portfolios to cover for at least a nonnegative proportion of the benchmark. The latter restriction, with the assumption of no arbitrage and a self financing portfolio, implies that the initial portfolio's value is nonnegative although allowing for negative portfolio positions. The benchmark that applies at time $T$, in this one-period monitoring setting, is known regardless if the benchmark is fixed, an Stock Index, or a high water mark (for example, with two monitoring dates it is not known in advance if the benchmark that applies is either the capital at the end of period one or the current benchmark value). In subsequent sections we relax and generalize some of these assumptions.

\subsection{Buying Calls and Puts on a Stock Index}

We first study the simple strategy of buying at time 0, a certain amount of European calls and puts on the Stock Index (assumed to be the benchmark) that mature at the end of the horizon and which do not necessarily have the same exercise price. Assume that the manager's objective is to maximize his expected fee and minimize the cost of using options to hedge his portfolio against underperformance with respect to the benchmark; we leave for subsequent sections the study of more complex objective functions. The optimal investment problem, without the constant term $f\left(\sum_{n \in N_{T}} \beta_{n} p_{n}\right)$, is

$$
\begin{aligned}
& \operatorname{Max}_{\left\{\theta, \epsilon_{0}, s\right\}} \quad\left(\alpha \sum_{n \epsilon N_{T}} \beta_{n} s_{n} p_{n}\right)-\beta_{0}\left(\epsilon_{0} \cdot V_{0}\right) \\
& \text { s.t. } \\
& Z_{0} \cdot \theta_{0}+\beta_{0}\left(\epsilon_{0} \cdot V_{0}\right)=\beta_{0} W_{0} \\
& Z_{n} \cdot\left(\theta_{n}-\theta_{a(n)}\right)=0, \forall n \epsilon N_{t} \forall t=1, \ldots, T \\
& Z_{n} \cdot \theta_{n}-\beta_{n} s_{n}+\beta_{n}\left(\epsilon_{0} \cdot V_{n}\right)=\beta_{n} B_{n}, \forall n \epsilon N_{T} \\
& Z_{n} \cdot \theta_{n} \geq 0, s_{n} \geq 0, \forall n \epsilon N_{T} \\
& \epsilon_{0} \geq \mathbf{0}
\end{aligned}
$$

where $\epsilon_{0} \equiv\left[\epsilon_{0}^{\text {Call }}, \epsilon_{0}^{\text {Put }}\right], V_{0} \equiv\left[C_{0}\left(1+T C_{\text {Call }}\right), P_{0}\left(1+T C_{P u t}\right)\right]$ and

$$
\begin{array}{ll}
s_{n} & : \text { The surplus over the benchmark } B_{n} \text { in scenario } n \epsilon N_{T} . \\
\epsilon_{0}^{\text {Call }}, \epsilon_{0}^{\text {Put }} & : \text { Call and Put options purchased, respectively. } \\
C_{0}, P_{0} & : \text { Call and Put option prices, respectively, at time } t=0 . \\
C_{n}, P_{n} & : \text { The payoff of the call and put options, respectively, } \\
& \text { on the stock index in scenario } n \epsilon N_{T} . \\
T C^{\text {Call }}, T C^{\text {Put }}: & \text { Transaction Costs when buying Calls and Puts respectively } \\
& \text { (as a percentage of the option's value). } \\
B_{n} & : \text { The Benchmark in scenario } n \epsilon N_{T} . \\
W_{0} & : \text { Initial Capital. }
\end{array}
$$


Furthermore,

$$
C_{n}=N \max \left\{S I_{n}-E^{C a l l}, 0\right\}, P_{n}=N \max \left\{E^{P u t}-S I_{n}, 0\right\}
$$

where:

$N \quad$ : The factor that applies (e.g. $N=100$ for an option on the SPX Index).

$S I_{n} \quad$ : The Stock Index level in scenario $n \in N_{T}$.

$E^{\text {Call }}$ : The exercise price of a Stock Index Call Option.

$E^{P u t}$ : The exercise price of a Stock Index Put Option.

The dual problem provides insights about the nature of the optimal strategies (feasibility of (1) is analyzed in section 4.5). The Lagrangian is

$$
\begin{aligned}
& L\left(\theta, \epsilon_{0}^{\text {Call }}, \epsilon_{0}^{\text {Put }}, s, y_{0}, y, x, \lambda, \mu, \eta_{\text {Call }}, \eta_{P u t}\right)= \\
& \left(\alpha \sum_{n \epsilon N_{T}} \beta_{n} s_{n} p_{n}\right)-\epsilon_{0}^{\text {Call }} \beta_{0} C_{0}\left(1+T C_{\text {Call }}\right)-\epsilon_{0}^{\text {Put }} \beta_{0} P_{0}\left(1+T C_{P u t}\right) \\
& -y_{0}\left(Z_{0} \cdot \theta_{0}+\epsilon_{0}^{\text {Call }} \beta_{0} C_{0}\left(1+T C_{\text {Call }}\right)+\epsilon_{0}^{\text {Put }} \beta_{0} P_{0}\left(1+T C_{P u t}\right)-\beta_{0} W_{0}\right) \\
& -\sum_{t=1}^{T} \sum_{n \epsilon N_{t}} y_{n}\left[Z_{n} \cdot\left(\theta_{n}-\theta_{a(n)}\right)\right] \\
& +\sum_{n \epsilon N_{T}} x_{n}\left(Z_{n} \cdot \theta_{n}-\beta_{n} s_{n}+\epsilon_{0}^{\text {Call }} \beta_{n} C_{n}+\epsilon_{0}^{\text {Put }} \beta_{n} P_{n}-\beta_{n} B_{n}\right) \\
& +\sum_{n \epsilon N_{T}} \lambda_{n}\left(Z_{n} \cdot \theta_{n}\right)+\sum_{n \epsilon N_{T}} \mu_{n} \beta_{n} s_{n}+\eta_{\text {Call }} \epsilon_{0}^{\text {Call }}+\eta_{P u t} \epsilon_{0}^{P u t}
\end{aligned}
$$

where:

$$
\begin{gathered}
\lambda_{n} \geq 0, \mu_{n} \geq 0, \forall n \in N_{T} \\
\eta_{\text {Call }} \geq 0, \eta_{\text {Put }} \geq 0,
\end{gathered}
$$

and $-y_{0},-y_{n}$ and $x_{n}$ are free variables since they correspond to equality constraints. The front signs have been chosen in purpose to give them later on a contextual interpretation. The Lagrangian is (see Appendix for proof)

$$
\begin{aligned}
& L\left(\theta, \epsilon_{0}^{\text {Call }}, \epsilon_{0}^{\text {Put }}, s, y_{0}, y, x, \lambda, \mu, \eta_{\text {Call }}, \eta_{P u t}\right)= \\
& y_{0} \beta_{0} W_{0}-\sum_{n \epsilon N_{T}} x_{n} \beta_{n} B_{n} \\
& +\sum_{n \epsilon N_{T}}\left(\alpha p_{n}+\mu_{n}-x_{n}\right) \beta_{n} s_{n}+\sum_{n \epsilon N_{T}}\left(\lambda_{n}+x_{n}-y_{n}\right) Z_{n} \cdot \theta_{n} \\
& -\sum_{t=0}^{T-1} \sum_{n \epsilon N_{t}}\left(y_{n} Z_{n}-\sum_{m \epsilon C(n)} y_{m} Z_{m}\right) \cdot \theta_{n} \\
& +\epsilon_{0}^{\text {Call }}\left[\sum_{n \epsilon N_{T}} x_{n} \beta_{n} C_{n}-\beta_{0} C_{0}\left(1+T C_{C a l l}\right)\left(1+y_{0}\right)+\eta_{C a l l}\right] \\
& +\epsilon_{0}^{\text {Put }}\left[\sum_{n \epsilon N_{T}} x_{n} \beta_{n} P_{n}-\beta_{0} P_{0}\left(1+T C_{P u t}\right)\left(1+y_{0}\right)+\eta_{P u t}\right]
\end{aligned}
$$

The dual constraints come from requiring that the factors of the primal variables are zero, and the dual objective function comes from all the Lagrangian terms that do not involve primal variables. The dual problem is 


$$
\begin{aligned}
& \operatorname{Min}_{\left\{y_{0}, y, x, \lambda, \mu, \eta_{\text {Call }}, \eta_{P u t}\right\}} \quad y_{0} \beta_{0} W_{0}-\sum_{n \epsilon N_{T}} x_{n} \beta_{n} B_{n} \\
& \text { s.t. } \\
& \alpha p_{n}+\mu_{n}-x_{n}=0, \forall n \epsilon N_{T} \\
& \lambda_{n}+x_{n}-y_{n}=0, \forall n \epsilon N_{T} \\
& \left(y_{n} Z_{n}-\sum_{m \epsilon C(n)} y_{m} Z_{m}\right)=0, \forall n \epsilon N_{t}, \forall t=0, \ldots, T-1 \\
& \sum_{n \epsilon N_{T}} x_{n} \beta_{n} C_{n}-\beta_{0} C_{0}\left(1+T C_{\text {Call }}\right)\left(1+y_{0}\right)+\eta_{\text {Call }}=0 \\
& \sum_{n \epsilon N_{T}} x_{n} \beta_{n} P_{n}-\beta_{0} P_{0}\left(1+T C_{P u t}\right)\left(1+y_{0}\right)+\eta_{P u t}=0 \\
& \lambda_{n} \geq 0, \mu_{n} \geq 0, \forall n \epsilon N_{T} \\
& \eta_{\text {Call }} \geq 0, \eta_{\text {Put }} \geq 0 .
\end{aligned}
$$

From the first two sets of restrictions of the dual

$$
x_{n}=\mu_{n}+\alpha p_{n} \geq \alpha p_{n}>0, \forall n \epsilon N_{T}
$$

and

$$
y_{n}=x_{n}+\lambda_{n} \geq x_{n}, \forall n \in N_{T}
$$

so

$$
y_{n} \geq x_{n} \geq \alpha p_{n}>0 .
$$

From the third set of restrictions of the dual problem and $Z_{n}^{0}=1 \forall n \epsilon N_{t}$, $\forall t=1, \ldots, T$,

$$
\begin{gathered}
y_{n}=\sum_{m \in C(n)} y_{m} \forall n \epsilon N_{t}, \forall t=1, \ldots, T \\
y_{0}=\sum_{n \in N_{T}} y_{n} .
\end{gathered}
$$

\section{Analysis and Interpretation of the Dual Problem}

1. From the dual objective function, and linear programming theory, the optimal dual variables $y_{0}^{*}$ and $x_{n}^{*}$ represent the marginal change of the optimal objective value with respect to the wealth $W_{0}$ and the benchmark $B_{n}$ respectively. Therefore, the larger in absolute value $y_{0}^{*}$ and $x_{n}^{*}$ are, the more sensitive the optimal strategy value is to a change in the initial wealth $W_{0}$ and the benchmark $B_{n}$.

2. The last two restrictions of the dual problem are equivalent to

$$
\begin{aligned}
& \left(1+y_{0}\right) \geq \sum_{n \in N_{T}} x_{n}\left(\frac{\beta_{n}}{\beta_{0}}\right)\left(\frac{C_{n}}{C_{0}}\right)\left(1+T C_{\text {Call }}\right)^{-1} \\
& \left(1+y_{0}\right) \geq \sum_{n \in N_{T}} x_{n}\left(\frac{\beta_{n}}{\beta_{0}}\right)\left(\frac{P_{n}}{P_{0}}\right)\left(1+T C_{P u t}\right)^{-1} .
\end{aligned}
$$

From 1, the right hand side of equations (8) and (9) can be interpreted as a weighted average return of each of the options (discounted by the transaction costs) that weighs up accordingly to the sensitivity of our optimal solution to a change in any of each of the possible benchmark values. 
3. From (5) and (7),

$$
\begin{gathered}
y_{0} \geq \sum_{n \epsilon N_{T}} x_{n} \\
\left(\Rightarrow\left(1+y_{0}\right) \geq\left(1+\sum_{n \epsilon N_{T}} x_{n}\right)\right) .
\end{gathered}
$$

4. From (8) - (10)

$$
\begin{aligned}
1+y_{0} \geq & \operatorname{Max}\left\{\sum_{n \epsilon N_{T}} x_{n}\left(\frac{\beta_{n}}{\beta_{0}}\right)\left(\frac{C_{n}}{C_{0}}\right)\left(1+T C_{C a l l}\right)^{-1},\right. \\
& \left.\sum_{n \epsilon N_{T}} x_{n}\left(\frac{\beta_{n}}{\beta_{0}}\right)\left(\frac{P_{n}}{P_{0}}\right)\left(1+T C_{P u t}\right)^{-1}, 1+\sum_{n \in N_{T}} x_{n}\right\}
\end{aligned}
$$

which together with the objective of minimizing the difference

$$
y_{0} \beta_{0} W_{0}-\sum_{n \in N_{T}} x_{n} \beta_{n} B_{n}
$$

yields

$$
\begin{aligned}
1+y_{0}^{*}= & \operatorname{Max}\left\{\sum_{n \epsilon N_{T}} x_{n}^{*}\left(\frac{\beta_{n}}{\beta_{0}}\right)\left(\frac{C_{n}}{C_{0}}\right)\left(1+T C_{\text {Call }}\right)^{-1},\right. \\
& \left.\sum_{n \epsilon N_{T}} x_{n}^{*}\left(\frac{\beta_{n}}{\beta_{0}}\right)\left(\frac{P_{n}}{P_{0}}\right)\left(1+T C_{P u t}\right)^{-1}, 1+\sum_{n \epsilon N_{T}} x_{n}^{*}\right\}
\end{aligned}
$$

If

$$
1+y_{0}^{*}=\sum_{n \in N_{T}} x_{n}^{*}\left(\frac{\beta_{n}}{\beta_{0}}\right)\left(\frac{C_{n}}{C_{0}}\right)\left(1+T C_{\text {Call }}\right)^{-1}
$$

or

$$
1+y_{0}^{*}=\sum_{n \in N_{T}} x_{n}^{*}\left(\frac{\beta_{n}}{\beta_{0}}\right)\left(\frac{P_{n}}{P_{0}}\right)\left(1+T C_{P u t}\right)^{-1}
$$

$y_{0}^{*}$ has already an interpretation (from 2.) as a weighted average return (discounted by transaction costs) of the Stock Index option (call or put). Since

$$
\sum_{n \in N_{T}} x_{n}^{*}=\sum_{n \in N_{T}} x_{n}^{*}\left(\frac{\beta_{n}}{\beta_{0}}\right)\left(\frac{1}{\frac{\beta_{n}}{\beta_{0}}}\right)
$$

then, if $1+y_{0}^{*}=1+\sum_{n \epsilon N_{T}} x_{n}^{*}, y_{0}^{*}$ and $\left\{x_{n}^{*}\right\}_{n \epsilon N_{T}}$ can be ,respectively, interpreted as the weighted average return from investing in a unit of the numeraire (that gives a return of $\left(\frac{\beta_{n}}{\beta_{0}}\right)^{-1}$ in scenario $n \epsilon N_{T}$ ), and a set of weighted scenario returns.

Therefore, in general, $y_{0}^{*}$ and the sequence $\left\{x_{n}^{*}\right\}_{n \in N_{T}}$ can be interpreted as the maximum weighted average return among the Stock Index options and the numeraire, and a set of weighted scenario returns respectively.

In general, since $y_{0}^{*}=\sum_{n \epsilon N_{T}} y_{n}^{*}$ (from equation (7)), the sequence $\left\{y_{n}^{*}\right\}_{n \in N_{T}}$ is interpreted as a set of scenario returns. 
5. If

$$
1+y_{0}^{*}=1+\sum_{n \in N_{T}} x_{n}^{*}
$$

is satisfied, it also implies, together with equations (5) and (7), that

$$
x_{n}^{*}=y_{n}^{*}, \forall n \epsilon N_{T}
$$

and hence, by linear complementarity,

$$
Z_{n} \cdot \theta_{n}^{*}>0, \forall n \in N_{T}
$$

i.e., in the optimum, the hedge fund manager requires a strictly positive terminal value on his portfolio for any possible scenario. The latter condition implies, given the no arbitrage assumption, that the initial portfolio value, $Z_{0} \cdot \theta_{0}$, is strictly positive, i.e.

$$
Z_{0} \cdot \theta_{0}^{*}>0
$$

6. From 2. and 4., the dual objective function

$$
y_{0} \beta_{0} W_{0}-\sum_{n \in N_{T}} x_{n} \beta_{n} B_{n}
$$

can be interpreted as the minimum difference between the return obtained from buying Stock Index options or the numeraire, and the weighted average benchmark.

7. From equations (5)-(7)

$$
q_{n} \equiv \frac{y_{n}}{y_{0}}>0, \forall n \in N_{T}
$$

defines a probability measure $Q$. Furthermore, the third set of restrictions of the dual problem (4) implies that $\left\{q_{n}\right\}_{n \in N_{T}}$ defines a martingale measure for $\left(Z_{n}\right)$. If the final value of the portfolio $Z_{n} \cdot \theta_{n}\left(n \in N_{T}\right)$ is unrestricted, the term

$$
\sum_{n \in N_{T}} \lambda_{n}\left(Z_{n} \cdot \theta_{n}\right)
$$

vanishes in equation (2) and hence $\sum_{n \epsilon n_{T}}\left(\lambda_{n}+x_{n}-y_{n}\right) Z_{n} \cdot \theta_{n}$ is replaced by

$$
\sum_{n \in N_{T}}\left(x_{n}-y_{n}\right) Z_{n} \cdot \theta_{n}
$$

in equation (3) and therefore, $x_{n}=y_{n}$ for all $n \in N_{T}$ and the dual objective function is

$$
y_{0}\left(\beta_{0} W_{0}-\sum_{n \in N_{T}}\left(\frac{y_{n}}{y_{0}}\right) \beta_{n} B_{n}\right)=y_{0}\left(\beta_{0} W_{0}-E^{Q}\left[\beta_{T} B_{T}\right]\right) .
$$

Hence, the optimal dual value

$$
y_{0}^{*}\left(\beta_{0} W_{0}-E^{Q^{*}}\left[\beta_{T} B_{T}\right]\right)
$$

can be interpreted as the weighted return over the remainder capital after discounting the initial hedge fund capital by the expected benchmark (under $Q^{*}$ ). 
8. Given 7, equation (5) provides us with a set of lower bounds for the dual variables $\left\{y_{n}\right\}_{n \in N_{T}}$ and hence, with a set of (scaled) lower bounds for the martingale probabilities $\left\{q_{n}\right\}_{n \in N_{T}}$. That is

$$
y_{0} q_{n} \geq \alpha p_{n} \forall n \in N_{T} .
$$

These lower bounds take more complex forms when other objective functions are considered later.

9. Finally, from equations (5) and (7)

$$
y_{0} \geq \alpha
$$

i.e., the return of investing in either calls, puts, or the numeraire must be at least the percentage of positive profits over the benchmark that the hedge fund manager receives.

We study optimal strategies under more realistic objective (utility) functions in subsequent sections. We now present a simple example and then discuss the strategy of selling Stock Index Options.

\section{An Example}

Consider one period with two scenarios for a portfolio with a bond (the numeraire) and a stock. The state prices of these two securities and the relevant stock index level, are shown in Figure 1 for each scenario.

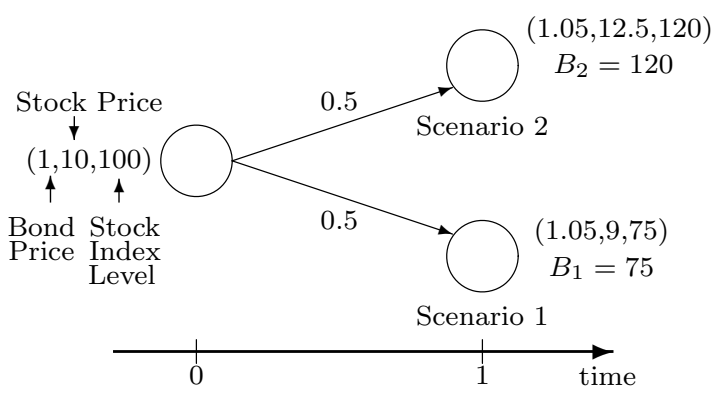

Fig. 1. State Prices and Benchmarks for each scenario.

Assume that the percentage of positive profits received by the hedge fund manager over the benchmark is $20 \%$ and that the Stock Index options have a factor $N=1$ (since we have scaled quantities), a common exercise price of 100 (i.e the options are at the money), and a fair value of

$$
\begin{aligned}
& C_{0}=\frac{1}{1.05}\left(q C_{2}+(1-q) C_{1}\right)=12.698 \\
& P_{0}=\frac{1}{1.05}\left(q P_{2}+(1-q) P_{1}\right)=7.937
\end{aligned}
$$


where:

$$
\begin{aligned}
q & =\left(\frac{105-75}{120-75}\right)=\frac{2}{3} \\
C_{2} & =20, \quad C_{1}=0 \\
P_{2} & =0, \quad P_{1}=25 .
\end{aligned}
$$

Assuming that initial capital is 95, the optimal strategy consists in investing the entire initial capital in the bond-stock portfolio

$$
\theta_{0}^{0}=-70 \text { (short position in bonds), } \theta_{0}^{1}=16.5 \text { (long position in the stock) }
$$

with an expected profit of

$$
\alpha \sum_{n \in N_{1}} \beta_{n} S_{n} p_{n}=\alpha \beta_{2} S_{2} p_{2}=1.214 .
$$

The optimal dual variable values are

$$
\begin{aligned}
y_{0}^{*}=0.2333, y_{1}^{*} & =0.1333, \quad x_{1}^{*}=0.1333, \\
y_{2}^{*} & =0.1, \quad \text { and } x_{2}^{*}=0.1 .
\end{aligned}
$$

Thus,

$$
\begin{aligned}
y_{0}^{*}= & 0.2333 \geq 0.2=\alpha \\
\sum_{n \epsilon N_{1}} x_{n}^{*}\left(\frac{\beta_{n}}{\beta_{0}}\right)\left(\frac{C_{n}}{C_{0}}\right) & =.15 \\
\sum_{n \in N_{1}} x_{n}^{*}\left(\frac{\beta_{n}}{\beta_{0}}\right)\left(\frac{P_{n}}{P_{0}}\right) & =0.3999 \\
\sum_{n \epsilon N_{1}} x_{n}^{*} & =0.2333 .
\end{aligned}
$$

Therefore

$$
1+y_{0}^{*}=1+\sum_{n \in N_{T}} x_{n}^{*}>\operatorname{Max}\left\{\sum_{n \in N_{1}} x_{n}^{*}\left(\frac{\beta_{n}}{\beta_{0}}\right)\left(\frac{C_{n}}{C_{0}}\right), \sum_{n \in N_{1}} x_{n}^{*}\left(\frac{\beta_{n}}{\beta_{0}}\right)\left(\frac{P_{n}}{P_{0}}\right)\right\} .
$$

The last two restrictions of the dual are not binding and therefore, by linear complementarity, $\left(\epsilon_{0}^{\text {Call }}\right)^{*}=\left(\epsilon_{0}^{\text {Put }}\right)^{*}=0$ so investing the entire initial hedge fund capital in the bond-stock portfolio is optimal.

Since $y_{1}^{*}=x_{1}^{*}$ and $y_{2}^{*}=x_{2}^{*}$, the interpretation 6 of the optimal objective value is a weighted return over the remainder capital of the initial hedge fund capital after discounting by the expected benchmark. Observe that

- The weighted return $y_{0}^{*}=23.33 \%$.

- The expected (discounted) Benchmark is

$$
\begin{aligned}
E^{Q^{*}}\left[\beta_{T} B_{T}\right] & =\left(\frac{1}{1.05}\right)\left[75 q_{1}^{*}+120 q_{2}^{*}\right] \\
& =\left(\frac{1}{1.05}\right)\left[75\left(\frac{y_{1}^{*}}{y_{0}^{*}}\right)+120\left(\frac{y_{2}^{*}}{y_{0}^{*}}\right)\right]=89.80 .
\end{aligned}
$$

- The remainder capital is

$$
\beta_{0} W_{0}-E^{Q^{*}}\left[\beta_{T} B_{T}\right]=95-89.80=5.20 .
$$


The optimal investment strategy gains $23.33 \%$ over the remainder capital of 5.20 .

The fact that the optimal investment strategy does not include Stock Index Options is not a mere coincidence. If the optimal investment strategy involves buying calls, i.e. $\left(\epsilon_{0}^{\text {Call }}\right)^{*}>0$ then, by linear complementarity,

$$
1+y_{0}^{*}=\sum_{n \in N_{T}} x_{n}^{*}\left(\frac{\beta_{n}}{\beta_{0}}\right)\left(\frac{C_{n}}{C_{0}}\right)\left(1+T C_{C a l l}\right)^{-1}
$$

which is equivalent to

$$
\beta_{0} C_{0}=\sum_{n \in N_{T}}\left(\frac{x_{n}^{*}}{1+y_{0}^{*}}\right)\left(\beta_{n}\right)\left(C_{n}\right)\left(1+T C_{C a l l}\right)^{-1}
$$

i.e., the option's value, $\beta_{0} C_{0}$, must necessarily satisfy (12). In our example,

$$
\beta_{0} C_{0}=12.698 \neq 1.5444=\sum_{n \in N_{1}}\left(\frac{x_{n}^{*}}{1+y_{0}^{*}}\right)\left(\beta_{n}\right)\left(C_{n}\right)\left(1+T C_{C a l l}\right)^{-1} .
$$

Analogously, if the optimal strategy involves buying puts, i.e. $\left(\epsilon_{0}^{P u t}\right)^{*}>0$, then

$$
\beta_{0} P_{0}=\sum_{n \in N_{T}}\left(\frac{x_{n}^{*}}{1+y_{0}^{*}}\right)\left(\beta_{n}\right)\left(P_{n}\right)\left(1+T C_{P u t}\right)^{-1}
$$

which, as it occurs with the call option, it is not satisfied in our example. Furthermore,

$$
\begin{aligned}
\sum_{n \epsilon N_{T}}\left(\frac{x_{n}^{*}}{1+y_{0}^{*}}\right)\left(\beta_{n}\right)\left(C_{n}\right)\left(1+T C_{C a l l}\right)^{-1} & \leq \sum_{n \epsilon N_{T}}\left(\frac{y_{n}^{*}}{1+y_{0}^{*}}\right)\left(\beta_{n}\right)\left(C_{n}\right)\left(1+T C_{C a l l}\right)^{-1} \\
& <\sum_{n \epsilon N_{T}}\left(\frac{y_{n}^{*}}{y_{0}^{*}}\right)\left(\beta_{n}\right)\left(C_{n}\right)\left(1+T C_{C a l l}\right)^{-1} \\
& =E^{Q^{*}}\left[\beta_{T} C_{T}\right]\left(1+T C_{C a l l}\right)^{-1} .
\end{aligned}
$$

Therefore, from (12) and (13), we have proved

Proposition 1 If $\left(\epsilon_{0}^{C a l l}\right)^{*}>0$ then

$$
\beta_{0} C_{0}\left(1+T C_{\text {Call }}\right)=\sum_{n \in N_{T}}\left(\frac{x_{n}^{*}}{1+y_{0}^{*}}\right) \beta_{n} C_{n}<E^{Q^{*}}\left[\beta_{T} C_{T}\right]
$$

where $Q^{*}=\left\{\frac{y_{n}^{*}}{y_{0}^{*}}\right\}_{n \epsilon N_{T}}$. Analogously, if $\left(\epsilon_{0}^{P u t}\right)^{*}>0$ then,

$$
\beta_{0} P_{0}\left(1+T C_{P u t}\right)=\sum_{n \in N_{T}}\left(\frac{x_{n}^{*}}{1+y_{0}^{*}}\right) \beta_{n} P_{n}<E^{Q^{*}}\left[\beta_{T} P_{T}\right]
$$

The valuation of contingent claims (in particular Stock Index options) consists essentially in finding a martingale measure $Q$ under which the value of the contingent claim is the expected value (under $Q$ ) of its discounted payoff. Proposition 1 means that buying calls or puts on an Stock Index is optimal then these must be strictly underpriced (under $Q^{*}$ ). Moreover, within this framework, King (2002) (p. 550) proved that the fair value of the contingent claim $V_{t}$ is

$$
\operatorname{Max}_{\{Q \epsilon \mathcal{Q}\}} E^{Q}\left[\beta_{T} V_{T}\right]
$$

where $V_{t}$ is the contingent's claim price at time $t$, and $\mathcal{Q}$ is the set of martingale measures of $\left\{V_{t}\right\}_{t=0, \ldots, T}$. Hence, 
Corollary 1 If $\left(\epsilon_{0}^{\text {Call }}\right)^{*}>0\left(\left(\epsilon_{0}^{\text {Call }}\right)^{*}>0\right)$ then

$$
\begin{aligned}
& \beta_{0} C_{0}\left(1+T C_{\text {Call }}\right)<\operatorname{Max}_{\{Q \in \mathcal{Q}\}} E^{Q}\left[\beta_{T} C_{T}\right] \\
& \left(\beta_{0} P_{0}\left(1+T C_{P u t}\right)<\operatorname{Max}_{\{Q \epsilon \mathcal{Q}\}} E^{Q}\left[\beta_{T} P_{T}\right]\right)
\end{aligned}
$$

In summary, when allowing for the purchase of Stock Index options, the optimal strategy will lead to a percentage (of at least $\alpha \%$ ) over the remainder of the initial hedge fund capital after discounting by the expected (discounted) benchmark and will include the purchase of options if these are strictly underpriced.

\subsection{Selling Calls and Puts on a Stock Index}

We now study the strategy of selling European calls and puts on a Stock Index at the beginning of the planning horizon and which mature at the end of this time horizon. The manager's objective is to maximize his expected variable fee and minimize the expected payout of the options sold. Inclusion of this expected payout term is done as a way of reducing the risk involved in the sale of options (other ways of modelling this risk are proposed in section 7). More advanced models are treated in subsequent sections. The hedge fund manager's optimal decision problem is

$$
\begin{aligned}
& \operatorname{Max}_{\left\{\theta, \bar{\epsilon}_{0}^{\text {Call }}, \bar{\epsilon}_{0}^{\text {Put }}, s\right\}} \quad\left(\alpha \sum_{n \epsilon N_{T}} \beta_{n} s_{n} p_{n}\right)-\left[\sum_{n \epsilon N_{T}}\left(\bar{\epsilon}_{0}^{C \text { Call }} \beta_{n} C_{n}+\bar{\epsilon}_{0}^{P u t} \beta_{n} P_{n}\right) p_{n}\right] \\
& \text { s.t. } \\
& Z_{0} \cdot \theta_{0}-\bar{\epsilon}_{0}^{\text {Call }} \beta_{0} \bar{C}_{0}\left(1+T C_{\text {Call }}\right)-\bar{\epsilon}_{0}^{\text {Put }} \beta_{0} \bar{P}_{0}\left(1+T C_{P u t}\right)=\beta_{0} W_{0} \\
& Z_{n} \cdot\left(\theta_{n}-\theta_{a(n)}\right)=0, \quad \forall n \in N_{t} \forall t=1, \ldots, T \\
& Z_{n} \cdot \theta_{n}-\beta_{n} s_{n}-\bar{\epsilon}_{0}^{\text {Call }} \beta_{n} C_{n}-\bar{\epsilon}_{0}^{\text {Put }} \beta_{n} P_{n}=\beta_{n} B_{n}, \quad \forall n \epsilon N_{T} \\
& Z_{n} \cdot \theta_{n} \geq 0, s_{n} \geq 0, \forall n \in N_{T} \\
& \bar{\epsilon}_{0}^{\text {Call }} \geq 0, \bar{\epsilon}_{0}^{\text {Put }} \geq 0 \text {. }
\end{aligned}
$$

where $\bar{\epsilon}_{0}^{\text {Call }}$ is the amount of call options on the Stock Index that are sold $\left(\bar{\epsilon}_{0}^{P u t}\right.$ is defined analogously), $\bar{C}_{0}$ the sale price and $\bar{P}_{0}$ the put option sale price. The dual of (14) provides important insights about the structure of the optimal strategies (feasibility of (14) is discussed in section 4.5). The Lagrangian is

$$
\begin{aligned}
& L\left(\theta, \bar{\epsilon}_{0}^{\text {Call }}, \bar{\epsilon}_{0}^{P u t}, s, y_{0}, y, x, \lambda, \mu, \eta_{\text {Call }}, \eta_{P u t}\right)= \\
& y_{0} \beta_{0} W_{0}-\sum_{n \epsilon N_{T}} x_{n} \beta_{n} B_{n} \\
& +\sum_{n \epsilon N_{T}}\left(\alpha p_{n}+\mu_{n}-x_{n}\right) \beta_{n} s_{n}+\sum_{n \epsilon N_{T}}\left(\lambda_{n}+x_{n}-y_{n}\right) Z_{n} \cdot \theta_{n} \\
& -\sum_{t=0}^{T-1} \sum_{n \epsilon N_{t}}\left(y_{n} Z_{n}-\sum_{m \epsilon C(n)} y_{m} Z_{m}\right) \theta_{n} \\
& +\bar{\epsilon}_{0}^{\text {Call }}\left[y_{0} \beta_{0} \bar{C}_{0}\left(1+T C_{C a l l}\right)-\sum_{n \epsilon N_{T}} x_{n} \beta_{n} C_{n}-\sum_{n \epsilon N_{T}}\left(\beta_{n} C_{n}\right) p_{n}+\eta_{\text {Call }}\right] \\
& +\bar{\epsilon}_{0}^{P u t}\left[y_{0} \beta_{0} \bar{P}_{0}\left(1+T C_{P u t}\right)-\sum_{n \epsilon N_{T}} x_{n} \beta_{n} P_{n}-\sum_{n \epsilon N_{T}}\left(\beta_{n} P_{n}\right) p_{n}+\eta_{P u t}\right] .
\end{aligned}
$$


The dual is

$$
\begin{aligned}
& \operatorname{Min}_{\left\{y_{0}, y, x, \lambda, \mu, \eta_{\text {Call }}, \eta_{P u t}\right\}} \quad y_{0} \beta_{0} W_{0}-\sum_{n \epsilon N_{T}} x_{n} \beta_{n} B_{n} \\
& \text { s.t. } \\
& \alpha p_{n}+\mu_{n}-x_{n}=0, \forall n \epsilon N_{T} \\
& \lambda_{n}+x_{n}-y_{n}=0, \forall n \epsilon N_{T} \\
& \left(y_{n} Z_{n}-\sum_{m \epsilon C(n)} y_{m} Z_{m}\right)=0, \forall n \epsilon N_{t}, \forall t=0, \ldots, T-1 \\
& y_{0} \beta_{0} \bar{C}_{0}\left(1+T C_{\text {Call }}\right)-\sum_{n \epsilon N_{T}} x_{n} \beta_{n} C_{n}-\sum_{n \epsilon N_{T}}\left(\beta_{n} C_{n}\right) p_{n}+\eta_{\text {Call }}=0 \\
& y_{0} \beta_{0} \bar{P}_{0}\left(1+T C_{P u t}\right)-\sum_{n \epsilon N_{T}} x_{n} \beta_{n} P_{n}-\sum_{n \epsilon N_{T}}\left(\beta_{n} P_{n}\right) p_{n}+\eta_{P u t}=0 \\
& \lambda_{n} \geq 0, \mu_{n} \geq 0, \forall n \epsilon N_{T} \\
& \eta_{\text {Call }} \geq 0, \eta_{P u t} \geq 0 .
\end{aligned}
$$

\section{Analysis and Interpretation of the Dual Problem}

The only difference between the dual problems (4) and (16) are the last two restrictions and thus we expect to inherit some of the interpretations of (4). Interpretations 1, 3, and $5-8$. apply exactly or almost exactly in the same manner, and 2 . and 4 . have similar counterparts as described below:

1. (2') The last two restrictions of the dual problem are equivalent to

$$
\begin{aligned}
y_{0} \leq & \sum_{n \epsilon N_{T}}\left[\left(\frac{\beta_{n}}{\beta_{0}}\right)\left(\frac{C_{n}}{C_{0}}\right)\left(1+T C_{\text {Call }}\right)^{-1}\right] p_{n}+ \\
& \sum_{n \epsilon N_{T}}\left[\left(\frac{\beta_{n}}{\beta_{0}}\right)\left(\frac{C_{n}}{C_{0}}\right)\left(1+T C_{\text {Call }}\right)^{-1}\right] x_{n} \\
y_{0} \leq & \sum_{n \epsilon N_{T}}\left[\left(\frac{\beta_{n}}{\beta_{0}}\right)\left(\frac{P_{n}}{P_{0}}\right)\left(1+T C_{\text {Put }}\right)^{-1}\right] p_{n}+ \\
& \sum_{n \epsilon N_{T}}\left[\left(\frac{\beta_{n}}{\beta_{0}}\right)\left(\frac{P_{n}}{P_{0}}\right)\left(1+T C_{P u t}\right)^{-1}\right] x_{n} .
\end{aligned}
$$

The right hand side of each of the previous restrictions can be interpreted as the sum of the expected option return (under $P$ ) and a weighted average return (the same described in interpretation 2 in section 4.2 ) that weighs up accordingly to a change in any of each of the possible benchmarks.

2. (4') Restrictions (17) and (10) imply that

$$
\begin{aligned}
\sum_{n \epsilon N_{T}} x_{n} \leq y_{0} \leq & \min \left\{\sum_{n \in N_{T}}\left[\left(\frac{\beta_{n}}{\beta_{0}}\right)\left(\frac{C_{n}}{C_{0}}\right)\left(1+T C_{\text {Call }}\right)^{-1}\right]\left(p_{n}+x_{n}\right),\right. \\
& \left.\sum_{n \in N_{T}}\left[\left(\frac{\beta_{n}}{\beta_{0}}\right)\left(\frac{P_{n}}{P_{0}}\right)\left(1+T C_{P u t}\right)^{-1}\right]\left(p_{n}+x_{n}\right)\right\}
\end{aligned}
$$

which together with the objective function of minimizing the difference

$$
y_{0} \beta_{0} W_{0}-\sum_{n \in N_{T}} x_{n} \beta_{n} B_{n}
$$

yields

$$
y_{0}^{*}=\sum_{n \in N_{T}} x_{n}^{*} .
$$

Therefore, as interpretation 5 in section $4.2, x_{n}^{*}=y_{n}^{*}$ for all $n \epsilon N_{T}$, thus $Z_{n} \cdot \theta_{n}^{*}>$ $0\left(n \in N_{T}\right)$ which, given the assumption of no arbitrage, lead us to a strictly positive initial portfolio's value. The latter fact does not exclude the possibility of including the 
sale of the Stock Index options as part of our optimal investment strategy. The next Proposition, analogous to Proposition 1, gives the conditions under which such sale is optimal.

Proposition 2 If $\left(\bar{\epsilon}_{0}^{\text {Call }}\right)^{*}>0$ then

$$
\begin{aligned}
\beta_{0} \bar{C}_{0}\left(1+T C_{\text {Call }}\right) & =E^{Q^{*}}\left[\beta_{T} C_{T}\right]+\left(\frac{1}{y_{0}^{*}}\right) E^{P}\left[\beta_{T} C_{T}\right] \\
& >E^{Q^{*}}\left[\beta_{T} C_{T}\right],
\end{aligned}
$$

where $Q^{*}=\left\{\frac{y_{n}^{*}}{y_{0}^{*}}\right\}_{n \in N_{T}}$. Analogously, if $\left(\bar{\epsilon}_{0}^{P u t}\right)^{*}>0$ then,

$$
\begin{aligned}
\beta_{0} \bar{P}_{0}\left(1+T C_{P u t}\right) & =E^{Q^{*}}\left[\beta_{T} P_{T}\right]+\left(\frac{1}{y_{0}^{*}}\right) E^{P}\left[\beta_{T} P_{T}\right] \\
& >E^{Q^{*}}\left[\beta_{T} P_{T}\right] .
\end{aligned}
$$

i.e., selling calls or puts on an Stock Index is optimal if these are strictly overpriced (under $Q^{*}$ ).

Proof: Assume that $\left(\epsilon_{0}^{\text {Call }}\right)^{*}>0$. Then, by linear complementarity,

$$
y_{0}^{*} \beta_{0} \bar{C}_{0}\left(1+T C_{\text {Call }}\right)=\sum_{n \in N_{T}}\left(\beta_{n} C_{n}\right) x_{n}^{*}+\sum_{n \in N_{T}}\left(\beta_{n} C_{n}\right) p_{n} .
$$

Optimality requires $y_{0}^{*}=\sum_{n \epsilon N_{T}} x_{n}^{*}$, hence $x_{n}^{*}=y_{n}^{*} \forall n \epsilon N_{T}$. Therefore

$$
\sum_{n \in N_{T}}\left(\beta_{n} C_{n}\right) x_{n}^{*}=\sum_{n \in N_{T}}\left(\beta_{n} C_{n}\right) y_{n}^{*} .
$$

Combining equations (18) and (19)

$$
y_{0}^{*} \beta_{0} \bar{C}_{0}\left(1+T C_{\text {Call }}\right)=\sum_{n \in N_{T}}\left(\beta_{n} C_{n}\right) y_{n}^{*}+\sum_{n \in N_{T}}\left(\beta_{n} C_{n}\right) p_{n} .
$$

Hence,

$$
\begin{aligned}
\beta_{0} \bar{C}_{0}\left(1+T C_{\text {Call }}\right) & =\sum_{n \epsilon N_{T}}\left(\beta_{n} C_{n}\right)\left(\frac{y_{n}^{*}}{y_{0}^{*}}\right)+\left(\frac{1}{y_{0}^{*}}\right) \sum_{n \epsilon N_{T}}\left(\beta_{n} C_{n}\right) p_{n} \\
& =E^{Q^{*}}\left[\beta_{T} C_{T}\right]+\left(\frac{1}{y_{0}^{*}}\right) E^{P}\left[\beta_{T} C_{T}\right]
\end{aligned}
$$

The Put option case is proved in an analogous manner.

Q.E.D.

It is crucial in Proposition 2 both, that condition $y_{0}^{*}=\sum_{n \epsilon N_{T}} x_{n}^{*}$ is satisfied, and the inclusion of the expected payout in the objective function.

In summary, when allowing for the sale of Stock Index options, the optimal strategy will lead to a percentage (of at least $\alpha \%$ ) over the remainder of the initial hedge fund capital after discounting by the expected (discounted) benchmark and will include the sale of options if these are strictly overpriced. 


\subsection{Buying and Selling Calls and Puts on an Stock Index}

The structure of optimal investment strategies when allowing for both the purchase and sale of Stock Index options with the same exercise price and time to maturity is straightforward following the discussion in the previous sections. The optimization model is

$$
\begin{aligned}
& \operatorname{Max}_{\left\{\theta, \epsilon_{0}^{\text {Call }}, \epsilon_{0}^{\text {Put }}, s\right\}}\left(\alpha \sum_{n \epsilon N_{T}} \beta_{n} s_{n} p_{n}\right)-\beta_{0}\left(\epsilon_{0} \cdot V_{0}\right)-\bar{\epsilon}_{0} \cdot\left(\sum_{n \epsilon N_{T}} \beta_{n} V_{n} p_{n}\right) \\
& \text { s.t. } \\
& Z_{0} \cdot \theta_{0}+\beta_{0}\left(\epsilon_{0} \cdot V_{0}-\bar{\epsilon}_{0} \cdot \bar{V}_{0}\right)=\beta_{0} W_{0} \\
& Z_{n} \cdot\left(\theta_{n}-\theta_{a(n)}\right)=0, \quad \forall n \epsilon N_{t} \forall t=1, \ldots, T \\
& Z_{n} \cdot \theta_{n}-\beta_{n} s_{n}+\beta_{n}\left(\epsilon_{0}-\bar{\epsilon}_{0}\right) \cdot V_{n}=\beta_{n} B_{n}, \forall n \epsilon N_{T} \\
& Z_{n} \cdot \theta_{n} \geq 0, s_{n} \geq 0, \forall n \epsilon N_{T} \\
& \epsilon_{0} \geq \mathbf{0}, \bar{\epsilon}_{0} \geq \mathbf{0}
\end{aligned}
$$

where

$$
\begin{gathered}
\epsilon_{0} \equiv\left[\epsilon_{0}^{\text {Call }}, \epsilon_{0}^{\text {Put }}\right], \bar{\epsilon}_{0} \equiv\left[\bar{\epsilon}_{0}^{\text {Call }}, \bar{\epsilon}_{0}^{\text {Put }}\right], V_{n} \equiv\left[C_{n}, P_{n}\right] \forall n \epsilon N_{T}, \\
V_{0} \equiv\left[\left(1+T C_{\text {Call }}\right) C_{0},\left(1+T C_{P u t}\right) P_{0}\right], \bar{V}_{0} \equiv\left[\left(1+T C_{\text {Call }}\right) \bar{C}_{0},\left(1+T C_{P u t}\right) \bar{P}_{0}\right] .
\end{gathered}
$$

The dual is

$$
\begin{aligned}
& \operatorname{Min}_{\left\{y_{0}, y, x\right\}} \quad y_{0} \beta_{0} W_{0}-\sum_{n \epsilon N_{T}} x_{n} \beta_{n} B_{n} \\
& \text { s.t. } \\
& \alpha p_{n}-x_{n} \leq 0, \forall n \epsilon N_{T} \\
& x_{n}-y_{n} \leq 0, \forall n \epsilon N_{T} \\
& \left(y_{n} Z_{n}-\sum_{m \epsilon C(n)} y_{m} Z_{m}\right)=0, \forall n \epsilon N_{t}, \forall t=0, \ldots, T-1 \\
& \sum_{n \epsilon N_{T}} x_{n} \beta_{n} C_{n}-\beta_{0} C_{0}\left(1+T C_{C a l l}\right)\left(1+y_{0}\right) \leq 0 \\
& \sum_{n \epsilon N_{T}} x_{n} \beta_{n} P_{n}-\beta_{0} P_{0}\left(1+T C_{P u t}\right)\left(1+y_{0}\right) \leq 0 \\
& y_{0} \beta_{0} \bar{C}_{0}\left(1+T C_{\text {Call }}\right)-\sum_{n \epsilon N_{T}} x_{n} \beta_{n} C_{n}-\sum_{n \epsilon N_{T}}\left(\beta_{n} C_{n}\right) p_{n} \leq 0 \\
& y_{0} \beta_{0} \bar{P}_{0}\left(1+T C_{P u t}\right)-\sum_{n \epsilon N_{T}} x_{n} \beta_{n} P_{n}-\sum_{n \epsilon N_{T}}\left(\beta_{n} P_{n}\right) p_{n} \leq 0 .
\end{aligned}
$$

Although Proposition 1 still holds for this case (under $Q^{*}$ ), Proposition 2 does not because $y_{0}^{*}=\sum_{n \in N_{T}} x_{n}^{*}$ does not necessarily hold.

Proposition 3 if $\left(\bar{\epsilon}_{0}^{\text {Call }}\right)^{*}>0\left(\left(\bar{\epsilon}_{0}^{\text {Put }}\right)^{*}>0\right)$ then

$$
\begin{gathered}
\beta_{0} \bar{C}_{0}\left(1+T C_{\text {Call }}\right) \leq E^{Q^{*}}\left[\beta_{T} C_{T}\right]+\left(\frac{1}{y_{0}^{*}}\right) E^{P}\left[\beta_{T} C_{T}\right] \\
\left(\beta_{0} \bar{P}_{0}\left(1+T C_{P u t}\right) \leq E^{Q^{*}}\left[\beta_{T} P_{T}\right]+\left(\frac{1}{y_{0}^{*}}\right) E^{P}\left[\beta_{T} P_{T}\right]\right)
\end{gathered}
$$


Proof: Assume $\left(\bar{\epsilon}_{0}^{\text {Call }}\right)^{*}>0$. By linear complementarity,

$$
y_{0}^{*} \beta_{0} \bar{C}_{0}\left(1+T C_{\text {Call }}\right)=\sum_{n \in N_{T}}\left(\beta_{n} C_{n}\right) x_{n}^{*}+\sum_{n \in N_{T}}\left(\beta_{n} C_{n}\right) p_{n} .
$$

Therefore,

$$
\beta_{0} \bar{C}_{0}\left(1+T C_{C a l l}\right)=\sum_{n \in N_{T}}\left(\beta_{n} C_{n}\right)\left(\frac{x_{n}^{*}}{y_{0}^{*}}\right)+\left(\frac{1}{y_{0}^{*}}\right) \sum_{n \in N_{T}}\left(\beta_{n} C_{n}\right) p_{n} .
$$

But,

$$
\begin{aligned}
1+y_{0}^{*} & =\operatorname{Max}_{n \epsilon N_{T}}\left\{1+\sum_{n \epsilon N_{T}} x_{n}^{*}, \sum_{n \epsilon N_{T}} x_{n}^{*}\left(\frac{\beta_{n}}{\beta_{0}}\right)\left(\frac{C_{n}}{C_{0}}\right), \sum_{n \epsilon N_{T}} x_{n}^{*}\left(\frac{\beta_{n}}{\beta_{0}}\right)\left(\frac{P_{n}}{P_{0}}\right)\right\} \\
& \geq 1+\sum_{n \epsilon N_{T}} x_{n}^{*} .
\end{aligned}
$$

Hence, $y_{0}^{*}=\sum_{n \epsilon N_{T}} y_{n}^{*} \geq \sum_{n \epsilon N_{T}} x_{n}^{*}$ and

$$
\beta_{0} \bar{C}_{0}\left(1+T C_{\text {Call }}\right) \leq \sum_{n \in N_{T}}\left(\beta_{n} C_{n}\right)\left(\frac{y_{n}^{*}}{y_{0}^{*}}\right)+\left(\frac{1}{y_{0}^{*}}\right) \sum_{n \in N_{T}}\left(\beta_{n} C_{n}\right) p_{n} .
$$

Q.E.D.

Therefore, when allowing for the purchase and sale of options, buying is optimal if options are strictly underpriced (under $Q^{*}$ ) and selling is optimal if their price is not larger than the expected payoff, under $Q^{*}$, plus a proportion $\frac{1}{y_{0}^{*}}$ of their expected payoff, under $P$.

\subsection{Feasibility of the two simple strategies}

Feasibility analysis leads us also to insights about the nature of the optimal investment strategies as well. We analyze the strategy of buying options on an Stock Index only since the sale case analysis is analogous, although with different interpretations and risk. Given (1), its feasibility reduces to validate the inequality

$$
\begin{aligned}
\beta_{0} W_{0} \geq & \operatorname{Min}_{\left\{\theta, \epsilon_{0}\right\}} Z_{0} \cdot \theta_{0}+\beta_{0}\left(\epsilon_{0} \cdot V_{0}\right) \\
& \text { s.t. } \\
& Z_{n} \cdot\left(\theta_{n}-\theta_{a(n)}\right)=0, \forall n \epsilon N_{t} \quad \forall t=1, \ldots, T \\
& Z_{n} \cdot \theta_{n}+\beta_{n} \epsilon_{0} \cdot V_{n}=\beta_{n} B_{n}, \forall n \epsilon N_{T} \\
& \epsilon_{0} \geq \mathbf{0}, Z_{n} \cdot \theta_{n} \geq 0 \forall n \epsilon N_{T} .
\end{aligned}
$$

The dual of the optimization problem on right hand side of inequality (22) is

$$
\begin{aligned}
& \operatorname{Max}_{\{x, q\}} \sum_{n \epsilon N_{T}} x_{n} \beta_{n} B_{n} \\
& \text { s.t. } \\
& q_{n} Z_{n}=\sum_{m \epsilon C(n)} q_{m} Z_{m}, \forall n \epsilon N_{t} \forall t=1, \ldots, T . \\
& q_{n} \geq 0, \forall n \epsilon N_{T} \\
& q_{0}=1 \\
& \beta_{0} V_{0} \leq \sum_{n \epsilon N_{T}} x_{n} \beta_{n} V_{n} \\
& q_{n} \geq x_{n} \geq 0, \forall n \epsilon N_{T}
\end{aligned}
$$


The first three restrictions imply that $Q \equiv\left\{q_{n}\right\}$ is a martingale measure of $\left\{Z_{t}\right\}_{t=0, \ldots, T}$. Hence, if $\mathcal{Q}$ is the set of all the martingale measures of $\left\{Z_{t}\right\}_{t=0, \ldots, T}$, the dual problem (23) becomes

$$
\begin{aligned}
& \operatorname{Max}_{\{x, Q \in \mathcal{Q}\}} \sum_{n \in N_{T}} x_{n} \beta_{n} B_{n} \\
& \text { s.t. } \\
& q_{n} \geq x_{n} \geq 0, \forall n \epsilon N_{T} \\
& \beta_{0} V_{0} \leq \sum_{n \in N_{T}} x_{n} \beta_{n} V_{n} .
\end{aligned}
$$

which is equivalent to

$$
\begin{aligned}
& \operatorname{Max}_{\{x, Q \in \mathcal{Q}\}} \sum_{n \in N_{T}} q_{n} \beta_{n} B_{n} \\
& \text { s.t. } \\
& \beta_{0} V_{0} \leq \sum_{n \in N_{T}} q_{n} \beta_{n} V_{n} .
\end{aligned}
$$

Therefore, we have proved

Proposition 4 Buying Stock Index options is feasible if and only if

$$
\begin{aligned}
\beta_{0} W_{0} \geq & \operatorname{Max}_{\{Q \in \mathcal{Q}\}} E^{Q}\left[\beta_{T} B_{T}\right] \\
& \text { s.t. } \\
& \beta_{0} V_{0} \leq E^{Q}\left[\beta_{T} V_{T}\right] .
\end{aligned}
$$

i.e., the initial capital of the hedge fund must be at least the maximum expected benchmark under martingale measures that value the option at a higher or equal price than its market value $\left(V_{0}\right)$.

Corollary 2 If buying Stock Index options only is feasible then

$$
\beta_{0} V_{0} \leq E^{Q^{*}}\left[\beta_{T} V_{T}\right]
$$

where $Q^{*}$ solves the right hand side of (24). That is, Stock Index options are not overpriced (under $Q^{*}$ ).

Also,

Proposition 5 Selling Stock Index options is feasible if and only if

$$
\begin{aligned}
\beta_{0} W_{0} \geq & \operatorname{Max}_{\{Q \in \mathcal{Q}\}} E^{Q}\left[\beta_{T} B_{T}\right] \\
& \text { s.t. } \\
& \beta_{0} \bar{V}_{0} \geq E^{Q}\left[\beta_{T} V_{T}\right] .
\end{aligned}
$$

i.e., the initial capital of the hedge fund must be at least the maximum expected benchmark under martingale measures that value the option at a lesser or equal price than its market value $\left(\bar{V}_{0}\right)$. 


\section{Other Utility functions}

We now study a more general class $\mathcal{U}$ of twice differentiable and strictly increasing concave utility functions than the probability-penalized weighted linear objective functions used in section 4. This class of utility functions are standard to incorporate risk aversion (Pratt (1964)). Differentiability assures that $U(\cdot)$ and $U^{\prime}(\cdot)$ are continuous and hence, if the feasible region is bounded (so a compact set), guarantees that the maximum is achieved. Strictly increasing assures that the dual variables define a probability measure.

\section{$\underline{\text { A Generic Problem }}$}

Let $U(\cdot)$ be a utility function in $\mathcal{U}$. We study, under the assumptions in 4.1, the optimal investment strategies when allowing for the purchase and sale of Stock Index options with the same exercise price and time to maturity (more general models are studied in section 7) and with the goal of maximizing the expected utility of the hedge fund manager's fee. The generic problem is

$$
\begin{aligned}
& \operatorname{Max} \sum_{n \epsilon N_{T}} U\left(\left[f+\alpha s_{n}\right] \beta_{n}\right) p_{n} \\
& \text { s.t. } \\
& Z_{0} \cdot \theta_{0}+\beta_{0}\left(\epsilon_{0} \cdot V_{0}-\bar{\epsilon}_{0} \cdot \bar{V}_{0}\right)=\beta_{0} W_{0} \\
& Z_{n} \cdot\left(\theta_{n}-\theta_{a(n)}\right)=0, \quad \forall n \epsilon N_{t} \forall t=1, \ldots, T \\
& Z_{n} \cdot \theta_{n}-\beta_{n} s_{n}+\beta_{n}\left(\epsilon_{0}-\bar{\epsilon}_{0}\right) \cdot V_{n}=\beta_{n} B_{n}, \quad \forall n \epsilon N_{T} \\
& Z_{n} \cdot \theta_{n} \geq 0, s_{n} \geq 0, \forall n \epsilon N_{T} \\
& \epsilon_{0} \geq \mathbf{0}, \bar{\epsilon}_{0} \geq \mathbf{0}
\end{aligned}
$$

The dual of this problem provides information about the optimal investment strategies. From standard nonlinear programming theory (e.g. Zangwill (1969) pp. 47 - 52) the dual of $(26)$ is

$$
\begin{aligned}
& \operatorname{Min}_{\left\{y_{0}, y, x\right\}} \quad\left(y_{0} \beta_{0} W_{0}-\sum_{n \epsilon N_{T}} x_{n} \beta_{n} B_{n}\right)+\sum_{n \epsilon N_{T}}\left(U\left(\left[f+\alpha s_{n}\right] \beta_{n}\right)-\alpha s_{n} U^{\prime}\left(\left[f+\alpha s_{n}\right] \beta_{n}\right)\right) p_{n} \\
& \text { s.t. } \\
& \alpha p_{n} U^{\prime}\left(\left[f+\alpha s_{n}\right] \beta_{n}\right)-x_{n} \leq 0, \forall n \epsilon N_{T} \\
& x_{n}-y_{n} \leq 0, \forall n \epsilon N_{T} \\
& \left(y_{n} Z_{n}-\sum_{m \epsilon C(n)} y_{m} Z_{m}\right)=0, \forall n \epsilon N_{t}, \forall t=0, \ldots, T-1 \\
& \sum_{n \epsilon N_{T}} x_{n} \beta_{n} V_{n}-\beta_{0} V_{0} y_{0} \leq \mathbf{0} \\
& -\sum_{n \epsilon N_{T}} x_{n} \beta_{n} V_{n}+\beta_{0} \bar{V}_{0} y_{0} \leq \mathbf{0} .
\end{aligned}
$$

Using the same (linear) restrictions of the model in section 4, we preserve several of its interpretations. For instance,

$$
y_{0}^{*}=\operatorname{Max}\left\{\sum_{n \in N_{T}} x_{n}^{*}, \sum_{n \in N_{T}} x_{n}^{*}\left(\frac{\beta_{n}}{\beta_{0}}\right)\left(\frac{C_{n}}{C_{0}}\right), \sum_{n \in N_{T}} x_{n}^{*}\left(\frac{\beta_{n}}{\beta_{0}}\right)\left(\frac{P_{n}}{P_{0}}\right)\right\}
$$

where $x_{n}^{*}$ and $y_{0}^{*}$ are scenario weights and an average return, respectively. Furthermore, given that $U(\cdot)$ is strictly increasing, the first two sets of restrictions in (27) imply that

$$
y_{n} \geq x_{n} \geq \alpha p_{n} U^{\prime}\left(\left[f+\alpha s_{n}\right] \beta_{n}\right)>0, \forall n \epsilon N_{T} .
$$


Therefore,

$$
q_{n} \equiv\left(\frac{y_{n}}{y_{0}}\right), \forall n \in N_{T}
$$

defines a probability measure for $\left(Z_{t}\right)_{t=0, \ldots, T}$. The dual objective function is formed by the sum of the (linear) objective function of the dual in section 4 ,

$$
y_{0} \beta_{0} W_{0}-\sum_{n \in N_{T}} x_{n} \beta_{n} B_{n},
$$

hereafter inheriting all the interpretations that are there pointed out, and the nonlinear term

$$
\sum_{n \in N_{T}}\left(U\left(\left[f+\alpha s_{n}\right] \beta_{n}\right)-\alpha s_{n} U^{\prime}\left(\left[f+\alpha s_{n}\right] \beta_{n}\right)\right) p_{n}
$$

which can be then interpreted as the expected nonlinear behavior of $U(\cdot)$ over the fee of the hedge fund manager.

Equation (29) is a generalization of equation (5) in section 4 and it defines a set of (scaled) lower bounds for the martingale probabilities $\left\{q_{n}\right\}_{n \in N_{T}}$. That is,

$$
\left\{\alpha p_{n} U^{\prime}\left(\left[f+\alpha s_{n}\right] \beta_{n}\right)\right\}_{n \in N_{T}}
$$

satisfy

$$
y_{0} q_{n} \geq \alpha p_{n} U^{\prime}\left(\left[f+\alpha s_{n}\right] \beta_{n}\right) \forall n \epsilon N_{T} .
$$

Equation (30) provides insights about the structure of the optimal investment strategy. It tell us that the relative weight that each scenario has as measured by the embedded martingale measure $\left\{q_{n}\right\}_{n \in N_{T}}$ (the higher the combination of probability and marginal utility over the hedge fund manager's fee for scenario $n \in N_{T}$, the greater $q_{n}$ tends to be). Given that $y_{0}=\sum_{n \epsilon N_{T}} y_{n}$ must be minimized, the optimal solution sets each $y_{n}$ as close as possible to $\alpha p_{n} U^{\prime}\left(\left[f+\alpha s_{n}\right] \beta_{n}\right)$ for every $n \in N_{T}$. Therefore, given that $U^{\prime}(\cdot)$ is nondecreasing, scenarios that lead to higher profits will tend to have a higher weight than scenarios that lead to lesser profits only if their natural or given (i.e. the $\left\{p_{n}\right\}_{n \in N_{T}}$ could be the subjective probability assessments of the manager) probabilities are significantly greater than the low-profit scenarios, i.e. if

$$
\alpha p_{n}^{H} U^{\prime}\left(\left[f+\alpha s_{n}^{H}\right] \beta_{n}\right) \geq \alpha p_{n}^{L} U^{\prime}\left(\left[f+\alpha s_{n}^{L}\right] \beta_{n}\right)
$$

where $p_{n}^{H}$ and $p_{n}^{L}$ are the high-profit and low-profit scenario probabilities, respectively. Or equivalently, given that $U(\cdot)$ is strictly increasing, if

$$
p_{n}^{H} \geq p_{n}^{L}\left[\frac{U^{\prime}\left(\left[f+\alpha s_{n}^{L}\right] \beta_{n}\right)}{U^{\prime}\left(\left[f+\alpha s_{n}^{H}\right] \beta_{n}\right)}\right]
$$

and hence, the significance between high-profit and low-profit scenario probabilities depending on the tradeoff probability factor

$$
\left[\frac{U^{\prime}\left(\left[f+\alpha s_{n}^{L}\right] \beta_{n}\right)}{U^{\prime}\left(\left[f+\alpha s_{n}^{H}\right] \beta_{n}\right)}\right] .
$$


Equation (30) can be reexpressed as

$$
y_{0} \geq \alpha\left(\frac{p_{n}}{q_{n}}\right) U^{\prime}\left(\left[f+\alpha s_{n}\right] \beta_{n}\right) \forall n \epsilon N_{T} .
$$

Hence

$$
y_{0}^{*} \geq \operatorname{Max}_{n \epsilon N_{T}}\left\{\alpha\left(\frac{p_{n}}{q_{n}}\right) U^{\prime}\left(\left[f+\alpha s_{n}\right] \beta_{n}\right)\right\} .
$$

Then, if

$$
\begin{aligned}
y_{0}^{*} & =\operatorname{Max}_{n \epsilon N_{T}}\left\{\alpha\left(\frac{p_{n}}{q_{n}}\right) U^{\prime}\left(\left[f+\alpha s_{n}\right] \beta_{n}\right)\right\} \\
& =\alpha\left(\frac{p_{n}}{q_{n}^{*}}\right) U^{\prime}\left(\left[f+\alpha s_{n}^{*}\right] \beta_{n}\right) \text { for } n \epsilon A_{T} \subseteq N_{T},
\end{aligned}
$$

it yields, by complementarity, that the hedge fund manager, following the optimal strategy, will receive a strictly positive profits for scenarios $\left(n \in A_{T}\right)$ that lead to a maximal combination of marginal utility and difference between their natural or given probability $p_{n}$ and their embedded probability $q_{n}$.

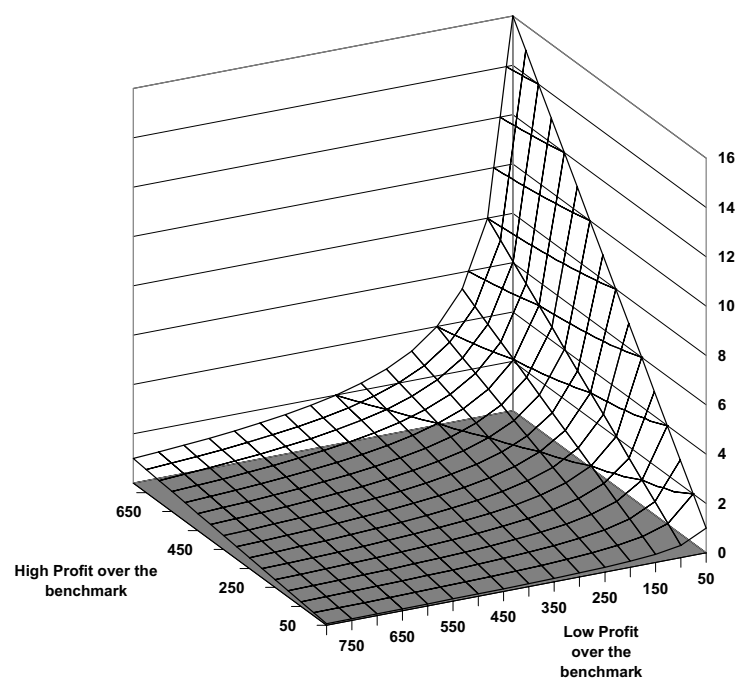

Fig. 2. Tradeoff Probability Factors for the Log utility function

To conclude, we state the conditions under which it is optimal to invest in options.

Proposition 6 (i) If $\left(\epsilon_{T_{1}}^{\text {Call }}\right)^{*}>0\left(\left(\epsilon_{T_{1}}^{P u t}\right)^{*}>0\right)$ then

$$
\begin{gathered}
\beta_{0} C_{0} \leq E^{Q^{*}}\left[\beta_{T} C_{T}\right] \\
\left(\beta_{0} P_{0} \leq E^{Q^{*}}\left[\beta_{T} P_{T}\right]\right),
\end{gathered}
$$


and if (ii) $\left(\bar{\epsilon}_{T_{1}}^{\text {Call }}\right)^{*}>0\left(\left(\bar{\epsilon}_{T_{1}}^{\text {Put }}\right)^{*}>0\right)$ then

$$
\begin{gathered}
\beta_{0} \bar{C}_{0} \leq E^{Q^{*}}\left[\beta_{T} C_{T}\right] \\
\left(\beta_{0} \bar{P}_{0} \leq E^{Q^{*}}\left[\beta_{T} P_{T}\right]\right)
\end{gathered}
$$

where $C_{0}\left(\bar{C}_{0}\right)$ and $P_{0}\left(\bar{P}_{0}\right)$ now denote the call and put prices including the transaction costs, and $Q^{*} \equiv\left\{\frac{y_{n}^{*}}{y_{0}^{*}}\right\}$ is a martingale measure for the process $\left(Z_{t}\right)_{t=0, \ldots, T}$.

i.e., buying or selling options is optimal if these are not overpriced under $Q^{*}$.

Proof: Only (i) is proved since the proof of (ii) is completely analogous.

(i) Assume $\left(\epsilon_{0}^{\text {Call }}\right)^{*}>0$. By complementarity,

$$
\sum_{n \in N_{T}} x_{n}^{*} \beta_{n} C_{n}=\beta_{0} C_{0} y_{0}^{*}
$$

But $y_{n}^{*} \geq x_{n}^{*} \forall n \epsilon N_{T}$, hence

$$
\sum_{n \in N_{T}} y_{n}^{*} \beta_{n} C_{n} \geq \sum_{n \in N_{T}} x_{n}^{*} \beta_{n} C_{n} \geq \beta_{0} C_{0} y_{0}^{*} .
$$

Therefore,

$$
E^{Q^{*}}\left[\beta_{T} C_{T}\right]=\sum_{n \in N_{T}} q_{n}^{*} \beta_{n} C_{n} \equiv \sum_{n \in N_{T}} \frac{y_{n}^{*}}{y_{0}^{*}} \beta_{n} C_{n} \geq \beta_{0} C_{0}
$$

It is analogously proved for put options.

\section{Q.E.D.}

It can be proved (see Proposition 7) that buying and selling simultaneously is optimal implies that the sale and purchase prices are equal.

Proposition 7 (i) If $\left(\epsilon_{0}^{\text {Call }}\right)^{*}>0$ and $\left(\bar{\epsilon}_{0}^{\text {Call }}\right)^{*}>0$ then

$$
\bar{C}_{0}=C_{0}
$$

(ii) Analogously, if $\left(\epsilon_{0}^{\text {Put }}\right)^{*}>0$ and $\left(\bar{\epsilon}_{0}^{\text {Put }}\right)^{*}>0$ then

$$
\bar{P}_{0}=P_{0}
$$

Proof: Only (i) is proved since proof of (ii) is analogous.

(i) Assume $\left(\epsilon_{0}^{\text {Call }}\right)^{*}>0$ and $\left(\bar{\epsilon}_{0}^{\text {Call }}\right)^{*}>0$. Then, by complementarity,

$$
\beta_{0} \bar{C}_{0}=\sum_{n \in N_{T}} x_{n}^{*} \beta_{n} C_{n}=\beta_{0} C_{0}
$$

Q.E.D. 
The latter result can also be obtained from feasibility of (27) and the assumption $\bar{V}_{0} \geq V_{0}$. Feasibility of (27) implies

$$
\beta_{0} \bar{V}_{0} y_{0} \leq \sum_{n \in N_{T}} x_{n} \beta_{n} V_{n} \leq \beta_{0} V_{0} y_{0}
$$

which combined with $\bar{V}_{0} \geq V_{0}$ leads to $\bar{V}_{0}=V_{0}$. These inequalities do not hold, for example, if the the options that are purchased and sold have different exercise price or time to maturity.

\section{Power and Log Utility Cases}

A popular family of utility functions that belong to $\mathcal{U}$ is the power utility function $U(x)=\left(\frac{B}{B-1}\right) x^{1-\frac{1}{B}}, B>0$. They imply that the investor possesses decreasing absolute risk aversion and constant relative risk aversion (see Huang et al (1988) and MacLean and Ziemba (1999). The nonlinear term of the objective function in (27) becomes

$$
\sum_{n \in N_{T}}\left[\frac{B}{B-1}\left(\left[f+\alpha s_{n}\right] \beta_{n}\right)^{1-\frac{1}{B}}-\alpha s_{n}\left(\left[f+\alpha s_{n}\right] \beta_{n}\right)^{-\frac{1}{B}}\right]
$$

and the tradeoff probability factors are

$$
\frac{\left(\left[f+\alpha s_{n}^{L}\right] \beta_{n}\right)^{-\frac{1}{B}}}{\left(\left[f+\alpha s_{n}^{H}\right] \beta_{n}\right)^{-\frac{1}{B}}} .
$$

Figure 2 displays the tradeoff probability factors for the most risky member of the power utility functions family: the $\log$ utility function $\left(U(x)=\log (x)=\lim _{B \rightarrow 1}\left(\frac{B}{B-1}\right) x^{1-\frac{1}{B}}\right)$. The lesser the low profits are, the higher the tradeoff probability factors are.

\section{The Multiple-Period Monitoring Case}

\subsection{Two-Period monitoring Case}

There are two monitoring dates, $T_{1}$ and $T_{2}$, in which the hedge fund capital is monitored against benchmarks. Assume that between monitoring dates the hedge fund manager's portfolio uses self-financing strategies and that at monitoring date $T_{1}$ a proportional amount $q$ (with $q \leq 100 \%$ ) of the profit over the benchmark, $s_{n}^{1}$, is discounted from the hedge fund capital. The discounted amount must cover at least for the variable fee of the hedge fund manager, i.e. $q \geq \alpha$. Rebalancing of the investment positions does not occur at $T_{1}$ but a period later at $T_{1}+1$, except for the cash received from stock index options and the profits over the benchmark at $T_{1}$ which are invested in the numeraire (e.g. invested in treasury bills or a deposit in the bank). Furthermore, our utility function is time-additive, i.e.

$$
U\left(\beta_{T_{1}}\left[f+\alpha s_{T_{1}}\right], \beta_{T_{2}}\left[f+\alpha s_{T_{2}}\right]\right)=U_{1}\left(\beta_{T_{1}}\left[f+\alpha s_{T_{1}}\right]\right)+U_{2}\left(\beta_{T_{2}}\left[f+\alpha s_{T_{2}}\right]\right)
$$

where $U_{t}(t=1,2) \in \mathcal{U}$. Two cases are considered:

- Fixed and Stock Index Benchmarks.

- High Water Marks. 


\section{Fixed and Stock Index Benchmarks}

Assume that the benchmark that applies is known in advance and is either a fixed value or a Stock Index (e.g. S\&P500) with certain probability distribution. The model is

$$
\begin{aligned}
& \operatorname{Max} \sum_{n \epsilon N_{T_{1}}} U_{1}\left(\left[f+\alpha s_{n}^{1}\right] \beta_{n}\right) p_{n}+\sum_{n \epsilon N_{T_{2}}} U_{2}\left(\left[f+\alpha s_{n}^{2}\right] \beta_{n}\right) p_{n} \\
& \text { s.t. } \\
& Z_{0} \cdot \theta_{0}+\beta_{0}\left(\epsilon_{0} \cdot V_{0}-\bar{\epsilon}_{0} \cdot \bar{V}_{0}\right)=\beta_{0} W_{0} \\
& Z_{n} \cdot\left(\theta_{n}-\theta_{a(n)}\right)=0, \quad \forall n \epsilon N_{t} \forall t=1, \ldots, T_{1} \\
& Z_{n} \cdot \theta_{n}-\beta_{n} s_{n}^{1}+\beta_{n}\left(\epsilon_{0}-\bar{\epsilon}_{0}\right) \cdot V_{n}^{0}=\beta_{n} B_{n}^{1}, \quad \forall n \epsilon N_{T_{1}} \\
& Z_{n} \cdot \theta_{n}+\beta_{n}\left(\epsilon_{T_{1}+1} \cdot V_{T_{1}+1, n}-\bar{\epsilon}_{T_{1}+1} \cdot \bar{V}_{T_{1}+1, n}\right)- \\
& {\left[Z_{n} \cdot \theta_{a(n)}+\beta_{n}\left(\epsilon_{0}-\bar{\epsilon}_{0}\right) \cdot V_{a(n)}^{0}\left(\frac{\beta_{a(n)}}{\beta_{n}}\right)-q \beta_{n} s_{a(n)}^{1}\left(\frac{\beta_{a(n)}}{\beta_{n}}\right)\right]=0, \quad \forall n \epsilon N_{T_{1}+1}} \\
& Z_{n} \cdot\left(\theta_{n}-\theta_{a(n)}\right)=0, \forall n \epsilon N_{t} \forall t=T_{1}+2, \ldots, T_{2} \\
& Z_{n} \cdot \theta_{n}-\beta_{n} s_{n}^{2}+\beta_{n}\left(\epsilon_{T_{1}+1}-\bar{\epsilon}_{T_{1}+1}\right) \cdot V_{n}^{T_{1}+1}=\beta_{n} B_{n}^{2}, \forall n \epsilon N_{T_{2}} \\
& Z_{n} \cdot \theta_{n} \geq 0, s_{n}^{1} \geq 0, \forall n \epsilon N_{T_{1}} \\
& Z_{n} \cdot \theta_{n} \geq 0, s_{n}^{2} \geq 0, \forall n \epsilon N_{T_{2}} \\
& \epsilon_{0} \geq \mathbf{0}, \bar{\epsilon}_{0} \geq \mathbf{0}, \epsilon_{T_{1}} \geq \mathbf{0}, \bar{\epsilon}_{T_{1}} \geq \mathbf{0} \\
& \text { where }
\end{aligned}
$$

$V_{t, n} \equiv\left[C_{t, n}, P_{t, n}\right]:$ Purchase prices in scenario $n, n \in N_{t}$.

$\bar{V}_{t, n} \equiv\left[\bar{C}_{t, n}, \bar{P}_{t, n}\right]:$ Sale prices in scenario $n, n \in N_{t}$.

$V_{n}^{t} \equiv\left[C_{n}^{t}, P_{n}^{t}\right]$ : Payoff in event $n$ ( $\epsilon N_{t}$ ) of an option bought at time $t$.

$\epsilon_{t} \equiv\left[\epsilon_{t}^{\text {Call }}, \epsilon_{t}^{\text {Put }}\right]:$ Amount of options purchased at time $t$.

$\bar{\epsilon}_{t} \equiv\left[\bar{\epsilon}_{t}^{\text {Call }}, \bar{\epsilon}_{t}^{\text {Put }}\right]$ : Amount of options sold at time $t$.

The dual is

$$
\begin{aligned}
& \operatorname{Min}_{\left\{y_{0}, y, x\right\}} \quad y_{0}^{1} \beta_{0} W_{0}-\sum_{n \epsilon N_{T_{1}}} x_{n}^{1} \beta_{n} B_{n}^{1}-\sum_{n \epsilon N_{T_{2}}} x_{n}^{2} \beta_{n} B_{n}^{2} \\
& +\sum_{n \epsilon N_{T_{1}}}\left(U_{1}\left(\left[f+\alpha s_{n}^{1}\right] \beta_{n}\right)-\alpha s_{n}^{1} U_{1}^{\prime}\left(\left[f+\alpha s_{n}^{1}\right] \beta_{n}\right)\right) p_{n} \\
& +\sum_{n \epsilon N_{T_{2}}}\left(U_{2}\left(\left[f+\alpha s_{n}^{2}\right] \beta_{n}\right)-\alpha s_{n}^{2} U_{2}^{\prime}\left(\left[f+\alpha s_{n}^{2}\right] \beta_{n}\right)\right) p_{n} \\
& \text { s.t. } \\
& \alpha p_{n} U_{1}^{\prime}\left(\left[f+\alpha s_{n}^{1}\right] \beta_{n}\right)-x_{n}^{1}-q y_{n}^{2} \leq 0, \forall n \epsilon N_{T_{1}} \\
& x_{n}^{1}-y_{n}^{1}+y_{n}^{2} \leq 0, \forall n \epsilon N_{T_{1}} \\
& \left(y_{n}^{1} Z_{n}-\sum_{m \epsilon C(n)} y_{m}^{1} Z_{m}\right)=0, \forall n \epsilon N_{t}, \forall t=0, \ldots, T_{1}-1 \\
& \sum_{n \epsilon N_{T_{1}}} x_{n}^{1} \beta_{n} V_{n}^{0}-\beta_{0} V_{0} y_{0}^{1}+\sum_{n \epsilon N_{T_{1}}} y_{n}^{2} \beta_{n} V_{n}^{0} \leq 0 \\
& \sum_{n \epsilon N_{T_{2}}} x_{n}^{2} \beta_{n} V_{n}^{T_{1}+1}-\sum_{n \epsilon N_{T_{1}+1}} y_{n}^{2} \beta_{n} V_{T_{1}+1, n} \leq 0 \\
& -\sum_{n \epsilon N_{T_{1}}} x_{n}^{1} \beta_{n} V_{n}^{0}+\beta_{0} \bar{V}_{0} y_{0}^{1}-\sum_{n \epsilon N_{T_{1}}} y_{n}^{2} \beta_{n} V_{n}^{0} \leq 0 \\
& -\sum_{n \epsilon N_{T_{2}}} x_{n}^{2} \beta_{n} V_{n}^{T_{1}+1}+\sum_{n \epsilon N_{T_{1}+1}} y_{n}^{2} \beta_{n} \bar{V}_{T_{1}+1, n} \leq 0 \\
& \alpha p_{n} U_{2}^{\prime}\left(\left[f+\alpha s_{n}^{2}\right] \beta_{n}\right)-x_{n}^{2} \leq 0, \forall n \epsilon N_{T_{2}} \\
& x_{n}^{2}-y_{n}^{2} \leq 0, \forall n \epsilon N_{T_{2}} \\
& \left(y_{n}^{2} Z_{n}-\sum_{m \epsilon C(n)} y_{m}^{2} Z_{m}\right)=0, \forall n \epsilon N_{t}, \forall t=T_{1}, \ldots, T_{2}-1
\end{aligned}
$$


where $y_{n}^{2} \equiv \sum_{m \epsilon C(n)} y_{m}^{2}, \forall n \epsilon N_{T_{1}}$.

From the dual, the conditions under which is optimal to invest in options at times 0 and $T_{1}$ are

Proposition 8 (i) If $\left(\epsilon_{0}^{\text {Call }}\right)^{*}>0\left(\left(\epsilon_{0}^{\text {Put }}\right)^{*}>0\right)$ then

$$
\begin{gathered}
\beta_{0} C_{0} \leq E^{Q_{1}^{*}}\left[\beta_{T_{1}} C_{T_{1}}^{0}\right] \\
\left(\beta_{0} P_{0} \leq E^{Q_{1}^{*}}\left[\beta_{T_{1}} P_{T_{1}}^{0}\right]\right)
\end{gathered}
$$

(ii) If $\left(\bar{\epsilon}_{0}^{\text {Call }}\right)^{*}>0\left(\left(\bar{\epsilon}_{0}^{\text {Put }}\right)^{*}>0\right)$ then

$$
\begin{gathered}
\beta_{0} \bar{C}_{0} \leq E^{Q_{1}^{*}}\left[\beta_{T_{1}} C_{T_{1}}^{0}\right] \\
\left(\beta_{0} \bar{P}_{0} \leq E^{Q_{1}^{*}}\left[\beta_{T_{1}} P_{T_{1}}^{0}\right]\right)
\end{gathered}
$$

where $Q_{1}^{*} \equiv\left\{\frac{\left(y_{n}^{1}\right)^{*}}{\left(y_{0}^{1}\right)^{*}}\right\}$ is a martingale measure for the process $\left(Z_{t}\right)_{t=0, \ldots, T_{1}}$.

Proof: (i) Assume $\left(\epsilon_{0}^{\text {Call }}\right)^{*}>0$. Then, by complementarity,

$$
\beta_{0} C_{0}\left(y_{0}^{1}\right)^{*}=\sum_{n \in N_{T_{1}}}\left(\left(x_{n}^{1}\right)^{*}+\left(y_{n}^{2}\right)^{*}\right) \beta_{n} C_{n}^{0} .
$$

From $y_{n}^{1} \geq x_{n}^{1}+y_{n}^{2} \geq x_{n}^{1}+q y_{n}^{2} \geq \alpha p_{n} U_{1}^{\prime}\left(f+\alpha s_{n}^{1}\right)>0 \forall n \epsilon N_{T_{1}}$ (the strict inequality from $U(\cdot)$ strictly increasing and differentiable), and $y_{0}^{1}=\sum_{n \in N_{T_{1}}} y_{n}^{1}$,

$$
\beta_{0} C_{0} \leq \sum_{n \in N_{T_{1}}}\left(\frac{\left(y_{n}^{1}\right)^{*}}{\left(y_{0}^{1}\right)^{*}}\right) \beta_{n} C_{n}^{0}=E^{Q_{1}^{*}}\left[\beta_{T_{1}} C_{T_{1}}^{0}\right]
$$

The martingale property of $Q_{1}^{*}$ comes from the third set of restrictions in (33).

(ii) By complementarity,

$$
\beta_{0} \bar{C}_{0}\left(y_{0}^{1}\right)^{*}=\sum_{n \in N_{T_{1}}}\left(\left(x_{n}^{1}\right)^{*}+\left(y_{n}^{2}\right)^{*}\right) \beta_{n} C_{n}^{0} .
$$

Therefore, by the properties of $y_{0}^{1}$ and $y_{n}^{1}$ discussed in (i),

$$
\beta_{0} \bar{C}_{0} \leq \sum_{n \in N_{T_{1}}}\left(\frac{\left(y_{n}^{1}\right)^{*}}{\left(y_{0}^{1}\right)^{*}}\right) \beta_{n} C_{n}^{0}=E^{Q_{1}^{*}}\left[\beta_{T_{1}} C_{T_{1}}^{0}\right] .
$$

Q.E.D. 
Proposition 9 (i) If $\left(\epsilon_{T_{1}+1}^{\text {Call }}\right)^{*}>0\left(\left(\epsilon_{T_{1}+1}^{\text {Put }}\right)^{*}>0\right)$ then

$$
\begin{gathered}
E^{Q_{2}^{*}}\left[\beta_{T_{2}} C_{T_{2}}^{T_{1}+1}\right] \geq E^{Q_{2}^{*}}\left[\beta_{T_{1}+1} C_{T_{1}+1}\right] \\
\left(E^{Q_{2}^{*}}\left[\beta_{T_{2}} P_{T_{2}}^{T_{1}+1}\right] \geq E^{Q_{2}^{*}}\left[\beta_{T_{1}+1} P_{T_{1}+1}\right]\right),
\end{gathered}
$$

and if (ii) $\left(\bar{\epsilon}_{T_{1}+1}^{\text {Call }}\right)^{*}>0\left(\left(\bar{\epsilon}_{T_{1}+1}^{\text {Put }}\right)^{*}>0\right)$ then

$$
\begin{gathered}
E^{Q_{2}^{*}}\left[\beta_{T_{2}} C_{T_{2}}^{T_{1}+1}\right] \geq E^{Q_{2}^{*}}\left[\beta_{T_{1}+1} \bar{C}_{T_{1}+1}\right] \\
\left(E^{Q_{2}^{*}}\left[\beta_{T_{2}} P_{T_{2}}^{T_{1}+1}\right] \geq E^{Q_{2}^{*}}\left[\beta_{T_{1}+1} \bar{P}_{T_{1}+1}\right]\right)
\end{gathered}
$$

where $Q_{2}^{*} \equiv\left\{\frac{\left(y_{n}^{2}\right)^{*}}{\left(y_{0}^{2}\right)^{*}}\right\}$ is a martingale measure for the process $\left(Z_{t}\right)_{t=T_{1}+1, \ldots, T_{2}}$, and $y_{0}^{2} \equiv$ $\sum_{n \in N_{T_{1}+1}} y_{n}^{2}$.

Proof: (i) Assume $\left(\epsilon_{T_{1}+1}^{\text {Call }}\right)^{*}>0$. Then, by complementarity,

$$
\sum_{n \in N_{T_{2}}}\left(x_{n}^{2}\right)^{*} \beta_{n} C_{n}^{T_{1}+1}=\sum_{n \in N_{T_{1}+1}}\left(y_{n}^{2}\right)^{*} \beta_{n} C_{T_{1}+1, n} .
$$

From $y_{n}^{2} \geq x_{n}^{2} \geq \alpha p_{n} U_{2}^{\prime}\left(f+\alpha s_{n}^{2}\right)>0 \forall n \epsilon N_{T_{2}}$ (the strict inequality from $U(\cdot)$ strictly increasing and differentiable),

$$
\sum_{n \in N_{T_{2}}}\left(y_{n}^{2}\right)^{*} \beta_{n} C_{n}^{T_{1}+1} \geq \sum_{n \in N_{T_{1}+1}}\left(y_{n}^{2}\right)^{*} \beta_{n} C_{T_{1}+1, n}
$$

Hence, dividing both sides of the inequality by $\left(y_{0}^{2}\right)^{*}$,

$$
E^{Q_{2}^{*}}\left[\beta_{T_{2}} C_{T_{2}}^{T_{1}+1}\right] \geq E^{Q_{2}^{*}}\left[\beta_{T_{1}+1} C_{T_{1}+1}\right]
$$

(ii) The proof is analogous to (i).

Q.E.D.

Remark 1. Both $E^{Q_{1}^{*}}[\cdot]$ and $E^{Q_{2}^{*}}[\cdot]$ are expected-valued operators based at time 0. For instance, $E^{Q_{2}^{*}}\left[\beta_{T_{1}} C_{T_{1}}\right]$ is the expected value (under $Q_{2}^{*}$ ) at time 0 of the (discounted) call option's price at time $T_{1}$ (and that expires at time $T_{2}$ ).

Propositions 8 and 9 state that both, purchasing and selling options at times 0 and $T_{1}+1$ is optimal if these are not overpriced under $Q_{1}^{*}$ and $Q_{2}^{*}$ respectively.

Besides the optimal investment conditions in terms of embedded martingale measures, the dual (33) provides other insights. The inequalities

$$
\begin{gathered}
y_{n}^{1} \geq x_{n}^{1}+y_{n}^{2} \geq x_{n}^{1}+q y_{n}^{2} \geq \alpha p_{n} U_{1}^{\prime}\left(\left[f+\alpha s_{n}^{1}\right] \beta_{n}\right) \forall n \epsilon N_{T_{1}} \\
y_{n}^{2} \geq x_{n}^{2} \geq \alpha p_{n} U_{2}^{\prime}\left(\left[f+\alpha s_{n}^{2}\right] \beta_{n}\right) \forall n \epsilon N_{T_{2}}
\end{gathered}
$$

imply that 


$$
\begin{array}{ll}
y_{0}^{1} q_{n}^{1}=y_{n}^{1} \geq \alpha p_{n} U_{1}^{\prime}\left(\left[f+\alpha s_{n}^{1}\right] \beta_{n}\right) & \forall n \epsilon N_{T_{1}} \\
y_{0}^{2} q_{n}^{2}=y_{n}^{2} \geq \alpha p_{n} U_{2}^{\prime}\left(\left[f+\alpha s_{n}^{2}\right] \beta_{n}\right) & \forall n \epsilon N_{T_{2}}
\end{array}
$$

which, as it occurs also in the one-period monitoring case of section 5 through equation (29), lead to (scaled) lower bounds for the martingale measures $\left\{q_{n}^{1}\right\}_{n \in N_{T_{1}}}$ and $\left\{q_{n}^{2}\right\}_{n \in N_{T_{2}}}$. Moreover, given that $y_{0}^{1}=\sum_{n \in N_{T_{1}}} y_{n}^{1}$ is minimized, $y_{n}^{1}$ gets as close as possible to $\alpha p_{n} U_{1}^{\prime}\left(\left[f+\alpha s_{n}^{1}\right]\right)$. Therefore, the relative weight that each scenario possesses depends on the combination of probability and marginal utility, and thus the concept of tradeoff probability factors developed in section 5 also applies here. Furthermore, minimizing $y_{n}^{1}$ implies, by equation (34), minimizing $y_{n}^{2}\left(n \in N_{T_{1}}\right)$ and hence minimizing $y_{n}^{2}\left(n \in N_{T_{2}}\right)$. Therefore, $y_{n}^{2}$ gets as close as possible to $\alpha p_{n} U_{2}^{\prime}\left(\left[f+\alpha s_{n}^{2}\right] \beta_{n}\right)$ for every $n \epsilon N_{T_{2}}$ and thus, the same insights apply at time $T_{2}$.

Thus we conclude that the embedded probabilities for scenarios at monitoring date $T_{1}$ do not depend on the utility over the fee gained at monitoring date $T_{2}$. This does not occur for the case of "high water marks".

\section{High Water Marks}

Assume that at the beginning of the time horizon $(t=0)$ there is already a preestablished benchmark $B_{n}^{1}$ for monitoring date $T_{1}$ (in scenario $n \epsilon N_{T_{1}}$ ) and that the benchmark $B_{n}^{2}$ for monitoring date $T_{2}$ (in scenario $n \in N_{T_{2}}$ ) is determined as the maximum of $B_{\hat{n}}^{1}$ and $S_{\hat{n}}^{1}$ where $\hat{n}$ is a event ancestor of event $n$. The latter condition is modelled by adding the restrictions

$$
\begin{aligned}
& -\beta_{n} B_{n}^{2}+\beta_{n} B_{n}^{1}+(1-\alpha) \beta_{n} s_{n}^{1}=0, \quad \forall n \epsilon N_{T_{1}} \\
& \beta_{n} B_{n}^{2}-\beta_{a(n)} B_{a(n)}^{2}=0, \forall n \epsilon N_{t} \quad \forall t=T_{1}+1, \ldots, T_{2}
\end{aligned}
$$

to formulation (32). The first set of restrictions in (36) update the benchmark at time $T_{1}$ by adding the adjusted profit $(1-\alpha) s_{n}^{1}$ (after discounting the variable fee paid to the hedge fund manager) to the previous benchmark $\beta_{n} B_{n}^{1}$ for every possible scenario at $T_{1}$. Once the benchmark has been updated, the second set of restrictions rolls over the updated benchmark value until time $T_{2}$ where the hedge fund capital is monitored again.

The inclusion of restrictions (36) into (32) implies:

the definition of additional dual variables $\left(l_{n}\right)$ of (33) that satisfy

$$
\begin{gathered}
l_{n}-\sum_{m \in C(n)} l_{m}=0, \forall n \in N_{t}, \quad t=T_{1}, \ldots, T_{2}-1 \\
-x_{n}^{2}+l_{n}=0, \quad \forall n \in N_{T_{2}},
\end{gathered}
$$

the modification of the first set of restrictions in (33)

$$
\alpha p_{n} U_{1}^{\prime}\left(\left[f+\alpha s_{n}^{1}\right] \beta_{n}\right)-x_{n}^{1}-q y_{n}^{2} \leq 0, \forall n \epsilon N_{T_{1}}
$$

by

$$
\alpha p_{n} U_{1}^{\prime}\left(\left[f+\alpha s_{n}^{1}\right] \beta_{n}\right)-x_{n}^{1}-q y_{n}^{2}+(1-\alpha) l_{n} \leq 0, \forall n \in N_{T_{1}},
$$


and the addition of

$$
\sum_{n \in N_{T_{2}}} l_{n} \beta_{n} B_{n}^{1}
$$

into the objective function of (33). Restriction (39) is crucial for expressing the optimal investment conditions in terms of implicit martingale measures and defining (scaled) lower bounds for these measures. For instance,

$$
\alpha p_{n} U_{1}^{\prime}\left(\left[f+\alpha s_{n}^{1}\right] \beta_{n}\right)+(1-\alpha) l_{n} \leq x_{n}^{1}+q y_{n}^{2} .
$$

But,

$$
x_{n}^{1}+q y_{n}^{2} \leq x_{n}^{1}+y_{n}^{2} \leq y_{n}^{1} \forall n \in N_{T_{1}},
$$

$U_{1}^{\prime}(\cdot)$ strictly increasing, (37) and (38), together with $x_{n}^{2} \geq \alpha p_{n} U_{2}^{\prime}\left(\left[f+\alpha s_{n}^{2}\right] \beta_{n}\right)$, imply

$$
0<\alpha p_{n} U_{1}^{\prime}\left(\left[f+\alpha s_{n}^{1}\right] \beta_{n}\right)+(1-\alpha) l_{n}, \forall n \in N_{T_{1}} .
$$

Therefore,

$$
0<\alpha p_{n} U_{1}^{\prime}\left(\left[f+\alpha s_{n}^{1}\right] \beta_{n}\right)+(1-\alpha) l_{n} \leq x_{n}^{1}+q y_{n}^{2} \leq y_{n}^{1} .
$$

Hence $Q_{1} \equiv\left\{\frac{y_{n}^{1}}{y_{0}^{1}}\right\}$ defines a probability measure. Propositions 8 and 9 hold for "high water marks" although with different embedded martingale measures $Q_{1}^{*}$ and $Q_{2}^{*}$. Indeed, $Q_{1}^{*}$ is in this case bounded from below. To see this observe that by applying (37) repeatedly,

$$
l_{n}=\sum_{m \in D^{T_{2}}(n)} l_{m}
$$

where $D^{T_{2}}(n)$ means any descent of node $n$ by time $T_{2}$. Then, by (38) and (35),

$$
\sum_{m \in D^{T_{2}}(n)} l_{m}=\sum_{m \in D^{T_{2}}(n)} x_{m}^{2} \geq \sum_{m \in D^{T_{2}}(n)} \alpha p_{m} U_{2}^{\prime}\left(\left[f+\alpha s_{m}^{2}\right] \beta_{m}\right)
$$

Therefore,

$$
l_{n} \geq \sum_{m \in D^{T_{2}}(n)} \alpha p_{m} U_{2}^{\prime}\left(\left[f+\alpha s_{m}^{2}\right] \beta_{m}\right)
$$

Hence, using (40) we conclude that $Q_{1}^{*}$ is bounded from below by

$$
\left\{\alpha p_{n}+(1-\alpha) \sum_{m \in D^{T_{2}}(n)} \alpha p_{m} U_{2}^{\prime}\left(\left[f+\alpha s_{m}^{2}\right] \beta_{m}\right)\right\}_{n \in N_{T_{1}}}
$$

i.e.

$$
y_{0}^{1} q_{n}^{1} \geq \alpha p_{n} U_{1}^{\prime}\left(\left[f+\alpha s_{n}^{1}\right] \beta_{n}\right)+(1-\alpha)\left(\sum_{m \in D^{T_{2}}(n)} \alpha p_{m} U_{2}^{\prime}\left(\left[f+\alpha s_{m}^{2}\right] \beta_{m}\right)\right) \forall n \epsilon N_{T_{1}}
$$

Therefore, given that $y_{n}^{1}\left(n \in N_{T_{1}}\right.$ ) is minimized, the embedded (martingale) probabilities $\left\{\left(q_{n}^{1}\right)^{*}\right\}_{n \in N_{T_{1}}}$ depend not only on the combination of natural or given probability and marginal utility at monitoring date $T_{1}$, but also on the accumulated (discounted) combinations of natural or given probability and marginal utility over the descendant scenarios of $n \in N_{T_{1}}$ by monitoring date $T_{2}$. The embedded (martingale) probabilities $\left\{\left(q_{n}^{2}\right)^{*}\right\}_{n \in N_{T_{2}}}$ depend, as in the case of fixed and stock index benchmarks, on the combinations of natural or given probability and marginal utility at $T_{2}$ only. 


\subsection{Multiple monitoring dates}

Once the two-period monitoring case is analyzed, it is straight forward to extend the results to the multi-period case. For instance, assume that there are $m$ monitoring dates $T_{1}, \ldots, T_{m}$ in which the hedge fund capital is monitored against certain benchmarks. As in the two-period case, assume that between monitoring dates the hedge fund manager's portfolio uses self-financing strategies and that at each monitoring date $T_{j}$, except for the last one (i.e., $j=1, \ldots, m-1$ ), a proportional amount $q$ (with $q \leq 100 \%$ ) of the profit over the benchmark, $s_{n}^{j}$, is discounted from the hedge fund capital. The discounted amount must cover for at least the variable fee of the hedge fund manager (i.e., $q \geq \alpha$ ). Rebalancing of the investment positions does not occur at $T_{j}$ but a period later at $T_{j}+1$, except for the cash received from stock index options and the profits over the benchmark at $T_{1}$ which are invested in the numeraire (e.g. invested in treasury bills or a deposit in the bank). Finally, our utility function over the entire horizon is assumed to be time-additive and each of its components to belong $\mathcal{U}$.

Regardless of the policy to determine the benchmarks, we have the following optimal conditions for buying or selling stock index options at time $T_{j}(j=1, \ldots, m-1)$

Theorem 1. (i) If $\left(\epsilon_{T_{j}+1}^{\text {Call }}\right)^{*}>0$ then

$$
E^{Q_{j+1}^{*}}\left[\beta_{T_{j+1}} C_{T_{j+1}}^{T_{j}+1}\right] \geq E^{Q_{j+1}^{*}}\left[\beta_{T_{j}+1} C_{T_{j}+1}\right]
$$

and (ii) If $\left(\bar{\epsilon}_{T_{j}+1}^{\text {Call }}\right)^{*}>0$ then

$$
E^{Q_{j+1}^{*}}\left[\beta_{T_{j+1}} C_{T_{j+1}}^{T_{j}+1}\right] \geq E^{Q_{j+1}^{*}}\left[\beta_{T_{j}+1} \bar{C}_{T_{j}+1}\right]
$$

where $Q_{j+1}^{*}=\left\{\frac{\left(y_{n}^{j+1}\right)^{*}}{\left(y_{0}^{*}\right)^{*}}\right\}_{n \in N_{T_{j+1}}}$

Proof: Only (i) is proved since (ii) is completely analogous. We consider two sets of benchmark policies.

Fixed or Stock Index Benchmarks

The dual restrictions are

$$
\begin{gathered}
\alpha p_{n} U_{j+1}^{\prime}\left(\left[f+\alpha s_{n}^{j+1}\right] \beta_{n}\right)-x_{n}^{j+1}-q y_{n}^{j+2} \leq 0 \forall n \in N_{T_{j+1}} \\
x_{n}^{j+1}+y_{n}^{j+2}-y_{n}^{j+1} \leq 0 \forall n \epsilon N_{T_{j+1}} \\
\sum_{n \in N_{T_{j+1}}}\left(x_{n}^{j+1}+y_{n}^{j+2}\right) \beta_{n} C_{n}^{T_{j}+1}-\sum_{n \in N_{T_{j}}} y_{n}^{j+1} \beta_{n} C_{T_{j}+1, n} \leq 0
\end{gathered}
$$

for $j=0, \ldots, m-2$, and

$$
\begin{gathered}
\alpha p_{n} U_{m}^{\prime}\left(\left[f+\alpha s_{n}^{m}\right] \beta_{n}\right)-x_{n}^{m} \leq 0 \forall n \epsilon N_{T_{m}} \\
x_{n}^{m}-y_{n}^{m} \leq 0 \forall n \epsilon N_{T_{m}}
\end{gathered}
$$




$$
\sum_{n \in N_{T_{m}}}\left(x_{n}^{m}\right) \beta_{n} C_{n}^{T_{m-1}+1}-\sum_{n \in N_{T_{m-1}}} y_{n}^{m} \beta_{n} C_{T_{m-1}+1, n} \leq 0
$$

for $T_{m-1}$.

Assume $\left(\epsilon_{T_{j}+1}^{\text {Call }}\right)^{*}>0$. Then,

Case 1: $j=0, \ldots, m-2$. Applying complementarity to (44)

$$
\sum_{n \in N_{T_{j+1}}}\left(\left(x_{n}^{j+1}\right)^{*}+\left(y_{n}^{j+2}\right)^{*}\right) \beta_{n} C_{n}^{T_{j}+1}=\sum_{n \in N_{T_{j}}}\left(y_{n}^{j+1}\right)^{*} \beta_{n} C_{T_{j}+1, n}
$$

From (42) and (43)

$$
0<\alpha p_{n} U_{j+1}^{\prime}\left(\left[f+\alpha s_{n}^{j+1}\right] \beta_{n}\right) \leq x_{n}^{j+1}+q y_{n}^{j+2} \leq x_{n}^{j+1}+y_{n}^{j+2} \leq y_{n}^{j+1}
$$

Hence,

$$
\sum_{n \in N_{T_{j+1}}} y_{n}^{j+1} \beta_{n} C_{n}^{T_{j}+1} \geq \sum_{n \in N_{T_{j}}} y_{n}^{j+1} \beta_{n} C_{T_{j}+1, n}
$$

and, dividing by $y_{0}^{j+1} \equiv \sum_{n \epsilon N_{T_{j}}} y_{n}^{j+1}$,

$$
E^{Q_{j+1}^{*}}\left[\beta_{T_{j+1}} C_{T_{j+1}}^{T_{j}}\right] \geq E^{Q_{j+1}^{*}}\left[\beta_{T_{j}} C_{T_{j}}\right]
$$

Case 2: The proof is analogous to case 1 .

The case of put options is proved in an analogous manner.

\section{$\underline{\text { High Water Marks }}$}

The relevant dual restrictions for high water marks are the same as for the two cases treated for fixed or stock index benchmarks except for (42) and (45) which are replaced, respectively, by

$\alpha p_{n} U_{j+1}^{\prime}\left(\left[f+\alpha s_{n}^{j+1}\right] \beta_{n}\right)-x_{n}^{j+1}-q y_{n}^{j+2}+(1-\alpha) l_{n}^{j+1} \leq 0, \quad \forall n \in N_{T_{j+1}}, \quad j=0, \ldots, m-2$ and,

$$
\alpha p_{n} U_{m}^{\prime}\left(\left[f+\alpha s_{n}^{m}\right] \beta_{n}\right)-x_{n}^{m}+(1-\alpha) l_{n}^{m} \leq 0, \forall n \in N_{T_{m}}
$$

where $l_{n}^{j+1}$ satisfies

$$
\begin{gathered}
l_{n}^{j+1}-\sum_{m \in C(n)} l_{m}^{j+1}=0, \quad \forall n \in N_{t} t=T_{j+1}, \ldots, T_{j+2}-1, j=0, \ldots, m-2 . \\
-x_{n}^{j+1}+l_{n}^{j+1}=0, \forall n \in N_{T_{j+1}}, j=1, \ldots, m-1 .
\end{gathered}
$$

The proof for this benchmark policy follows the same structure used for the case of fixed or stock index benchmarks.

Q.E.D.

Although Theorem 1 holds regardless of the benchmark policy, the embedded martingale measures $\left\{Q_{j}\right\}$ are not the same. For fixed or stock index benchmarks the $Q_{j}$ 's depends only on the period utility $U_{j}$ while for high water marks, the $Q_{j}$ 's depend on $U_{j}, \ldots, U_{m}$. 


\section{More Advanced Models}

The models here incorporate risk management features and other extensions not treated in sections $4-6$. For instance, these models include risk management of underperformance (with respect to a benchmark), stock index options, and other risky factors such as liquidity and short selling. To simplify, we focus on the single monitoring date case and depart from the basic set of constraints

$$
\begin{aligned}
& Z_{0} \cdot \theta_{0}+\beta_{0}\left(\epsilon_{0} \cdot V_{0}-\bar{\epsilon}_{0} \cdot \bar{V}_{0}\right)=\beta_{0} W_{0} \\
& Z_{n} \cdot\left(\theta_{n}-\theta_{a(n)}\right)=0, \quad \forall n \epsilon N_{t} \forall t=1, \ldots, T \\
& Z_{n} \cdot \theta_{n}-\beta_{n} s_{n}+\beta_{n}\left(\epsilon_{0}-\bar{\epsilon}_{0}\right) \cdot V_{n}=\beta_{n} B_{n}, \forall n \epsilon N_{T} \\
& Z_{n} \cdot \theta_{n} \geq 0, s_{n} \geq 0, \forall n \epsilon N_{T} \\
& \epsilon_{0} \geq \mathbf{0}, \bar{\epsilon}_{0} \geq \mathbf{0} .
\end{aligned}
$$

\subsection{Underperformance Risk Management}

Regardless of the strategy followed by the manager, the risk of underperformance with respect to a benchmark is a major issue. Therefore, appropriate risk measures should be included in the objective function of the hedge fund manager to take into account for this risk. An important class of measures in that sense are the downside-risk aversion measures (Fishburn (1977)) that penalize on the shortfall of the portfolio relative to a given benchmark $B_{T}$. Within the class of downside-risk aversion measures, there is a widely applied subclass known as lower partial moment class (Bawa and Lindenberg (1977)). In this subclass the penalization of the shortfall is done through partial (statistical) moments of the shortfall and hence defining risk measures of the form

$$
R_{\gamma}\left(B_{T}\right) \equiv E\left[\operatorname{Max}\left(B_{T}-W_{T}, 0\right)^{\gamma}\right],
$$

where $\gamma \geq 0$ and $W_{T}$ represents the wealth at time $T$. The values of $\gamma$ determine the weight that the investor gives to small or large deviations. The larger the $\gamma$, the more the investor cares for larger deviations and viceversa. Moreover, for particular values of $\gamma, R_{\gamma}\left(B_{T}\right)$ represents measures such as shortfall probability $(\gamma=0)$, expected shortfall $(\gamma=1)$, and downside variance $(\gamma=2)$.

$R_{\gamma}\left(B_{T}\right)$ can be combined with the goal of maximizing the hedge fund manager's fee through the objective function

$$
\operatorname{Max} E\left[\left(f+\alpha s_{T}\right) \beta_{T}\right]-A R_{\gamma}\left(B_{T} \beta_{T}\right)
$$

where $A$ is a nonnegative constant that determines the risk aversion of the hedge fund manager or equivalently the tradeoff between expected profit and underperformance risk. In our scenario framework, (49) when $\gamma=1$ (expected shortfall) is

$$
\operatorname{Max} \sum_{n \in N_{T}}\left[f+\alpha s_{n}\right] \beta_{n} p_{n}-A \sum_{n \in N_{T}}\left(S F_{n} \beta_{n}\right) p_{n}
$$

where $S F_{n}$ is the shortfall relative to the benchmark $B_{n}$ in scenario $n \epsilon N_{T}$, and which, besides nonnegativity restrictions, satisfies the (modified) set of constraints

$$
Z_{n} \cdot \theta_{n}-\beta_{n} s_{n}+\beta_{n}\left(\epsilon_{0}-\bar{\epsilon}_{0}\right) \cdot V_{n}^{0}+\beta_{n} S F_{n}=\beta_{n} B_{n} \forall n \epsilon N_{T},
$$

and $A>\alpha$ (to assure feasibility). The latter conditions on $A$ and $S F_{n}\left(n \in N_{T}\right)$ together with the objective function (50) enforces 


$$
S F_{n}^{*}=\operatorname{Max}\left(\beta_{n} B_{n}-\left[Z_{n} \cdot \theta_{n}+\beta_{n}\left(\epsilon_{0}-\bar{\epsilon}_{0}\right) V_{n}^{0}\right], 0\right) .
$$

To see this, observe that if $Z_{n} \cdot \theta_{n}^{*}+\beta_{n}\left(\epsilon_{0}^{*}-\left(\bar{\epsilon}_{0}\right)^{*}\right) V_{n}^{0}>\beta_{n} B_{n}$ then $-s_{n}^{*}+S F_{n}^{*}<0$ and hence $s_{n}^{*}>S F_{n}^{*}$. The objective function is

$$
\begin{gathered}
\left(\sum_{m \epsilon N_{T}} f \beta_{m} p_{m}\right)+\alpha \sum_{m \epsilon N_{T}}\left(Z_{m} \cdot \theta_{m}^{*}+\beta_{m}\left(\epsilon_{0}^{*}-\left(\bar{\epsilon}_{0}\right)^{*}\right) V_{m}^{0}-\beta_{m} B_{m}\right) p_{m} \\
+(\alpha-A) \sum_{m \epsilon N_{T}} S F_{m}^{*} p_{m} .
\end{gathered}
$$

Therefore, since $(\alpha-A)<0$, the latter is maximized when $S F_{n}^{*}=0$. When $Z_{n} \cdot \theta_{n}^{*}+$ $\beta_{n}\left(\epsilon_{0}^{*}-\left(\bar{\epsilon}_{0}\right)^{*}\right) V_{n}^{0}<\beta_{n} B_{n}$ it can be proven that $s_{n}^{*}=0$ (hence $S F_{n}^{*}=\beta_{n} B_{n}-Z_{n} \cdot \theta_{n}^{*}-$ $\left.\beta_{n}\left(\epsilon_{0}^{*}-\left(\bar{\epsilon}_{0}\right)^{*}\right) V_{n}^{0}\right)$.

The inclusion of the set of variables $\left(S F_{n}\right)_{n \in N_{T}}$ not only extends the feasible region defined by (48) but also adds the restrictions

$$
x_{n} \leq A p_{n} \forall n \in N_{T} .
$$

to the dual associated to (48) (See (27) in section 5).

Model (50) subject to (48) (with the modified restriction (51)) possesses the same optimality conditions for option investment stated in Proposition 6. Nevertheless, the addition of the dual restriction (52) gives us more structure, and hence more insights, about the optimal solutions. For instance, if $s_{n}^{*}>0$ and $S F_{n}^{*}>0$ imply, by complementarity, $x_{n}^{*}=\alpha p_{n}$ and $x_{n}^{*}=A p_{n}$, respectively. That is $x_{n}^{*}$ will be an indicator function of profits and shortfalls, i.e.

$$
x_{n}^{*}=\left\{\begin{array}{l}
\alpha p_{n} \text { if } s_{n}^{*}>0 \\
A p_{n} \text { if } S F_{n}^{*}>0
\end{array} \quad \forall n \in N_{T} .\right.
$$

\subsection{Stock Index Option Risk Management}

Besides underperformance, hedge fund managers must also consider appropriate risk management of any trading strategy that is implemented in their pursue of superior returns. We propose here some risk measures and their modelling for the strategy of buying and selling stock index options.

Buying options is much less risky than selling options since their maximum loss is bounded. At worst, the premium paid for the option is lost if the option expires out of the money. Therefore, an appropriate risk measure for the purchase of a stock index option, and any option in general, is

$$
\delta\left(V_{0} \beta_{0}\right)
$$

where $\delta$ is the probability that the option expires out of the money ( $\delta$ can be computed once the scenarios have been set up and therefore it is known at the moment of solving the model), and $V_{0}$ is the option's price (call or put). Hence, assuming homogeneity of the risk measure (i.e. the double position have double the risk, see Artzner et al (1999)), a model incorporates a tradeoff between profit and risk over the purchased options in our framework is

$$
\operatorname{Max} \sum_{n \in N_{T}}\left[f+\alpha s_{n}\right] \beta_{n} p_{n}-\epsilon_{0}\left[\delta\left(V_{0} \beta_{0}\right)\right]
$$

subject to (48). The inclusion of $\epsilon_{0}\left[\delta\left(V_{0} \beta_{0}\right)\right]$ yields 
Proposition 10 If $\left(\epsilon_{0}^{\text {Call }}\right)^{*}>0$ then

$$
\beta_{0} C_{0}=\sum_{n \in N_{T}}\left(\frac{x_{n}^{*}}{\delta+y_{0}^{*}}\right) \beta_{n} C_{n}<E^{Q^{*}}\left[\beta_{T} C_{T}\right]
$$

$$
\begin{aligned}
& \text { where } Q^{*}=\left\{\frac{y_{n}^{*}}{y_{0}^{*}}\right\}_{n \in N_{T}} \text {. Analogously, if }\left(\epsilon_{0}^{P u t}\right)^{*}>0 \text { then, } \\
& \qquad \beta_{0} P_{0}=\sum_{n \in N_{T}}\left(\frac{x_{n}^{*}}{\delta+y_{0}^{*}}\right) \beta_{n} P_{n}<E^{Q^{*}}\left[\beta_{T} P_{T}\right] .
\end{aligned}
$$

Proof: The proof is analogous to that of Proposition 1.

i.e., buying options in the current model is optimal if these are underpriced (under $Q^{*}$ )

Remark 2. The underpricing of Proposition 10 is less restrictive than the one derived from Proposition 1. The underpricing threshold is higher for the current model than for the simple strategy analyzed in section 4.2. The scenario weights in Proposition 1 are

$$
\frac{x_{n}^{*}}{1+y_{0}^{*}} \text {. }
$$

Hence, since

$$
\frac{x_{n}^{*}}{1+y_{0}^{*}} \leq \frac{x_{n}^{*}}{\delta+y_{0}^{*}} \forall n \in N_{T},
$$

the current model assigns to each scenario more weight than the simple strategy of section 4.2 does.

Selling options implies taking a position in which losses are, in principle, unbounded and hence risk management is crucial. Hedging the risk of sold options is usually carried out through either the implementation of a portfolio of assets that replicates the payout of the option or the purchase of an option of the same characteristics (i.e. same expiry date and exercise price). The latter is partially implicit in the basic formulation (48) since it allows for buying and selling options of the same type although the model does not enforce the purchase of options if stock index options are sold. On the other hand, perfect replication of the payout, which is also implicit in formulation (48), is not always possible since our framework allows for incomplete markets. Therefore, it is imperative to include risk measures into the basic formulation We addressed this problem in section 4.3 by including the term

$$
\sum_{n \in N_{T}} \beta_{n} V_{n}^{0} p_{n}
$$

as a measure of risk for sold options. We now address risk management for sold options in a broader sense, for a class of risk measures that satisfy two properties: homogeneity and a priori determination (i.e., once the scenarios have been set up, the risk measure can be determined). Two members of this class are Value-at-Risk (VaR) and Conditional Value-at-Risk (CVaR) (See Mina and Yi Xiao (2001)) VaR is the maximum loss expected under a certain confidence level and CVaR is defined as the expected loss given that the loss has surpassed VaR. VaR is the most widely used risk measure and CVaR, although 
more conservative than VaR, is a more robust risk measure. For instance, Artzner et al (1999) prove that CVaR is a coherent risk measure (while VaR is not), Rockafellar and Uryasev (2000 and 2000a) prove that CVaR is convex and continuous, and Rockafellar et al (2000b) prove that CVaR is an expectation-bounded risk measure (while VaR is not). Other convex risk measures which place more weight on losses are discussed in Rockafellar and Ziemba (2000) and Cariño and Ziemba (1998). If it is assumed that VaR is computed at the confidence level of $(1-\gamma) \%$, the tradeoff between profit and risk over sold options can be modelled as

$$
\operatorname{Max} \sum_{n \in N_{T}}\left[f+\alpha s_{n}\right] \beta_{n} p_{n}-\bar{\epsilon}_{0}(\gamma V a R)
$$

subject to (48) if VaR is used or

$$
\operatorname{Max} \sum_{n \in N_{T}}\left[f+\alpha s_{n}\right] \beta_{n} p_{n}-\bar{\epsilon}_{0}(\gamma \operatorname{CondVaR})
$$

subject to (48) if CVaR is used. Using duality yields

Proposition 11 if $\left(\bar{\epsilon}_{0}^{\text {Call }}\right)^{*}>0\left(\left(\bar{\epsilon}_{0}^{\text {Put }}\right)^{*}>0\right)$ then

(i) under model (55),

$$
\begin{gathered}
\beta_{0} \bar{C}_{0}=\sum_{n \in N_{T}}\left(\frac{x_{n}^{*}}{y_{0}^{*}}\right) \beta_{n} C_{n}+\left(\frac{1}{y_{0}^{*}}\right)(\eta V a R), \text { and } \\
\left(\beta_{0} \bar{P}_{0}=\sum_{n \in N_{T}}\left(\frac{x_{n}^{*}}{y_{0}^{*}}\right) \beta_{n} P_{n}+\left(\frac{1}{y_{0}^{*}}\right)(\eta V a R)\right)
\end{gathered}
$$

(ii) under model (56),

$$
\begin{gathered}
\beta_{0} \bar{C}_{0}=\sum_{n \in N_{T}}\left(\frac{x_{n}^{*}}{y_{0}^{*}}\right) \beta_{n} C_{n}+\left(\frac{1}{y_{0}^{*}}\right)(\eta C V a R) . \\
\left(\beta_{0} \bar{P}_{0}=\sum_{n \in N_{T}}\left(\frac{x_{n}^{*}}{y_{0}^{*}}\right) \beta_{n} P_{n}+\left(\frac{1}{y_{0}^{*}}\right)(\eta C V a R)\right) .
\end{gathered}
$$

Proof: Only (i) is proved since (ii) is completely analogous.

Assume $\left(\bar{\epsilon}_{0}^{C a l l}\right)^{*}>0$. Then, by complementarity,

$$
y_{0}^{*} \beta_{0} \bar{C}_{0}=\sum_{n \in N_{T}} x_{n}^{*} \beta_{n} C_{n}+\eta V a R .
$$

Therefore,

$$
\beta_{0} \bar{C}_{0}=\sum_{n \in N_{T}}\left(\frac{x_{n}^{*}}{y_{0}^{*}}\right) \beta_{n} C_{n}+\left(\frac{1}{y_{0}^{*}}\right)(\eta V a R) .
$$

Q.E.D. 


\subsection{Other Extensions}

7.3.1. Restrictions on the positions of the portfolio None of the models studied impose any restrictions on the asset positions within the portfolio. For example, liquidity or short selling restrictions might be important risk management decisions and/or particular hedge fund policies, and therefore these constraints should be considered. The modelling of these type of restrictions requires the constraints

$$
\theta_{n} \geq b_{n} \forall n \in N_{t}, t=0, \ldots, T \text {. }
$$

which leads, with (48), to the dual restrictions

$$
\begin{gathered}
y_{n} Z_{n}-\sum_{m \in C(n)} y_{m} Z_{m}-\lambda_{n}=0 \forall n \in N_{t}, t=0, \ldots, T-1 \\
x_{n}-y_{n}+\lambda_{n}^{0} \leq 0 \forall N_{T}, \\
\lambda_{n} \geq \mathbf{0}, \forall n \in N_{t}, t=0, \ldots, T-1,
\end{gathered}
$$

and the addition to the dual objective function of the term

$$
-\sum_{n \in N_{T}} \lambda_{n} b_{n}
$$

Although $y_{n} \geq 0 \forall n \in N_{t}, t=0, \ldots, T,\left\{q_{n} \equiv \frac{y_{n}}{y_{0}}\right\}_{n \in N_{T}}$ is no longer a probability measure on $\left(\Omega, F_{T}\right)$. Moreover, the inclusion of restrictions on the positions of the portfolio destroys the martingale property of the $\left(Z_{t}\right)$.

7.3.2. Further extensions The model (48) assumes that the hedge fund manager buys and sells options with the same payoff. A natural extension is to allow for buying and selling options with different payoffs and/or different times to maturity. For instance, the initial capital constraint is still of the form

$$
Z_{0} \cdot \theta_{0}+\beta_{0}\left(\epsilon_{0} \cdot V_{0}-\bar{\epsilon}_{0} \cdot \bar{V}_{0}\right)=\beta_{0} W_{0}
$$

but now $\epsilon_{0}=\left(\epsilon_{0}^{1}, \ldots, \epsilon_{0}^{N}\right)$ and $\bar{\epsilon}_{0}=\left(\bar{\epsilon}_{0}^{1}, \ldots, \epsilon_{0}^{M}\right)$, where $\epsilon_{0}^{j}\left(\bar{\epsilon}_{0}^{j}\right)$ is the purchase (sale) price of the $j^{\text {th }}$ option (European calls or puts) for $j=1, \ldots, N(j=1, \ldots, M)$. Self-financing restrictions are

$$
Z_{n} \cdot\left(\theta_{n}-\theta_{a(n)}\right)-\beta_{n}\left(\epsilon_{0}^{t} \cdot V_{n}^{t}-\bar{\epsilon}_{0}^{t} \cdot \bar{V}_{n}^{t}\right)=0, \forall n \epsilon N_{t}
$$

where $\epsilon_{0}^{t}\left(\bar{\epsilon}_{0}^{t}\right)$ is the vector of positions of the option purchased (sold) at time 0 and which mature at time $t$, and $V_{n}^{t}\left(\bar{V}_{n}^{t}\right)$ is the vector of corresponding payoffs. The Benchmark restriction is

$$
Z_{n} \cdot \theta_{n}-\beta_{n} s_{n}+\beta_{n}\left(\epsilon_{0}^{T} \cdot V_{n}^{T}-\bar{\epsilon}_{0}^{T} \cdot \bar{V}_{n}^{T}\right)=\beta_{n} B_{n}, \forall n \epsilon N_{T}
$$

Duality can be applied then to get insights on the structure of optimal investments as well.

To conclude, we emphasize that the present models take into account different features of the hedge fund manager problem with the idea of simplifying the analysis. We can combine these models to develop more sophisticated ones. 


\section{Conclusions}

This paper develops a framework for a variety of models for hedge fund managers that encompasses the primary goal of maximizing their fees while attempting to obtain superior returns in any market condition using options strategies and appropriate risk management.

The framework has several advantages. First, it allows for a broad class of distributions including the fat-tailed distributions of real asset prices. Second, the framework is applicable to both incomplete and complete markets. Third, the mathematics used here are more accessible and understandable than other approaches.

This paper obtains a general description of the optimal solution for the hedge fund manager's problem for various models with different policies for determining benchmarks and useful interpretations of the corresponding dual problems; explicit and calculable optimal conditions for stock index option investments for several models in terms of embedded martingale probabilities; and explicit and calculable relationships between embedded probabilities and hedge fund manager preferences which allow assessment of the relative weight of future scenarios for a particular investors and hedge fund managers with different preferences.

The framework can be applied to general option-like compensation problems other than the hedge fund manager problem which could include problems such as the compensation of a corporate manager that controls firm leverage or the compensation of a trader at a security firm.

Although the theoretical insights developed remain valid regardless of the number of scenarios, the evaluation of the optimal investment conditions for the various models requires an efficient algorithm to compute the optimal values of their corresponding dual problems. Successful implementation requires good scenario generation procedures.

More complex models can be analyzed. For instance, models that incorporate more sophisticated and realistic risk measures such as dynamic risk measures, or strategies other than self-financing ones, or other risky factors such as the liquidation of the hedge fund. 


\section{References:}

1. Ackermann C., McEnally R., and Ravenscraft, The Performance of Hedge Funds: Risk, Return and Incentives, Journal of Finance, vol. 54 (1999), pp. 833 - 874.

2. Artzner P., Delbaen F., Eber J., and Heath D., Coherent measures of risk, Mathematical Finance, vol.9 No. 3 pp. 203 - 228.

3. Baxter M. and Rennie A., Financial Calculus, An Introduction to Financial Derivatives, Cambridge University Press, 1996.

4. Bawa V. and Lindenberg E, Capital market equilibrium in a mean-lower partial moment framework, Journal of Financial Economics 5, pp. 189 - 200.

5. Bazaraa M.S., Jarvis J.J., and Sherali H.D., Linear Programming and Network Flows, John Wiley and Sons, 1990.

6. Billingsley P., Probability and Measure, John Wiley and Sons, 1979.

7. Birge J.R. and Louveaux F., Introduction to Stochastic Programming, Springer Series in Operations Research, 1997.

8. de Brouwer G., Hedge Funds in Emerging Markets, Cambridge University Press, 2001.

9. Campbell J.Y., Lo A.W., and MacKinlay A.C., The Econometrics of Financial Markets, Princeton University Press, 1997.

10. Cariño D.R. and Ziemba W.T., Formulation of the Rusell-Yasuda financial planning model, Operations Research, July-August 1998, pp. 433 - 449.

11. Carpenter J., Does Option Compensation Increase Managerial Risk Appetite ?, The Journal of Finance, Oct. 2002, pp. 2311 - 2331.

12. Duffie D., Dynamic Asset Pricing Theory, Princeton University Press, 2001 (third edition).

13. Dybvig P.H., Farnsworth H.K., and Carpenter J.N., Porfolio Performance and Agency, Working Paper, Washington University in Saint Louis, 2000.

14. Eichengreen B., Donald Mathieson D., Chadha B., Jansen A., Kodres L., and Sharma S., Hedge Funds and Financial Market Dynamics, IMF, 1998.

15. Fishburn P., Mean-risk analysis with risk associated with below-target returns, American Economic Review, pp. 116 - 125.

16. Fung W., and Hsieh D., Empirical Characteristics of Dynamic Trading Strategies: The Case of Hedge Funds, Review of Financial Studies, vol. 10 (1997), pp. 275 - 302.

17. Goetzmann W.N., Ingersoll J. Jr., and Ross S. A.,High Water Marks and Hedge Fund Management Contracts, Journal of Finance, 2003, forthcoming.

18. Heinkel R. and Stoughton N.M., The Dynamics of Portfolio Management Contracts, Review of Financial Studies, vol. 7 (1994), pp. 351 - 387.

19. Huang C. and Litzenberger R.H., Foundations for Financial Economics, North-Holland, 1988.

20. Jorion P., Risk Management Lessons from Long-Term Capital Management,European Financial Management 6 (2000), pp. 277 - 300.

21. Karatzas I. and Shreve S.E., Brownian Motion and Stochastic Calculus, Springer, 1991 (second edition).

22. King A.L., Duality and Martingales: A Stochastic Programming Perspective on Contingent Claims, Mathematical Programming (Series B), 91 (2002), pp. 543 - 562.

23. Kouwenberg R., Do Hedge Funds Add Value to a Passive Portfolio: Correcting for Non Normal Returns and Disappearing Funds, Journal of Asset Management, vol. 3, pp. $361-382$.

24. Kouwenberg R. and Ziemba W.T., Incentives and Risk Taking in Hedge Funds, mimeo, Erasmus University (2003).

25. Liu J. and Longstaff F.A., Losing Money on Arbitrages: Optimal Dynamic Portfolio Choice in Markets with Arbitrage Opportunities, Working Paper, UCLA, June 2000. 
26. Loewenstein M. and Willard G.A.,Convergence Trades and Liquidity: A model for Hedge Funds, Working Paper,MIT, Jan. 2000.

27. MacLean L.C. and Ziemba W.T., Growth versus Security Tradeoffs in Dynamic Investment Analysis in Stochastic Programming: State of the Art 1998, eds. R. J-B Wets and W.T. Ziemba, Balzer Science Publishers, Annals of Operations Research, 1999, 193 226.

28. Maug E. and Naik. N., Herding and Delegated Portfolio Management: The Impact of Relative Performance Evaluation on Asset Allocation, Working Paper, London Business School, 1995.

29. Mina J. and Yi Xiao J., Return to RiskMetrics: The Evolution of the Standard, RiskMetrics, 2001.

30. Pratt J., Risk aversion in the small and in the large, Econometrica vol. 32, pp. 122 136.

31. Rockafellar R.T. and Uryasev S., Optimization of conditional value-at-risk, Journal of Risk, vol. 2 pp. 21 - 41, 2000.

32. Rockafellar R.T. and Uryasev S., Conditional value-at-risk for general loss distributions, Journal of Banking and Finance, vol. 26 pp. 1443 - 1471, 2002a.

33. Rockafellar R.T., Uryasev S., and Zabarankin M., Deviation measures in risk analysis and optimization, Research Report \# 2002-7, Risk Management and Financial Engineering Lab, Center of Applied Optimization, Department of Industrial and Systems Engineering, University of Florida, 2002b.

34. Rockafellar R.T. and Ziemba W.T., Modified Risk Measures and Acceptance sets, Mimeo, University of Washington, 2000.

35. Zangwill W.I., Nonlinear Programming, A Unified Approach, Prentice-Hall, 1969.

36. Ziemba W.T., The Stochastic Programming Approach to Asset, Liability and Wealth Management, AIMR, 2003. 


\section{Appendix}

\section{Equivalence between equations (2) and (3)}

The equivalence between equations (2) and (3) strives in showing that

$$
\begin{array}{r}
y_{0}\left(Z_{0} \cdot \theta_{0}\right)+\sum_{t=1}^{T} \sum_{n \epsilon N_{t}} y_{n}\left[Z_{n} \cdot\left(\theta_{n}-\theta_{a(n)}\right)\right]= \\
\sum_{n \in N_{T}} y_{n}\left(Z_{n} \cdot \theta_{n}\right)+\sum_{t=0}^{T-1} \sum_{n \epsilon N_{t}}\left(y_{n} Z_{n}-\sum_{m \epsilon C(n)} y_{m} Z_{m}\right) \cdot \theta_{n}
\end{array}
$$

Proof:

$$
\begin{array}{r}
y_{0}\left(Z_{0} \cdot \theta_{0}\right)+\sum_{t=1}^{T} \sum_{n \epsilon N_{t}} y_{n}\left[Z_{n} \cdot\left(\theta_{n}-\theta_{a(n)}\right)\right]= \\
\sum_{t=0}^{T} \sum_{n \epsilon N_{t}} y_{n}\left(Z_{n} \cdot \theta_{n}\right)-\sum_{t=1}^{T} \sum_{n \epsilon N_{t}} y_{n} Z_{n} \cdot \theta_{a(n)}= \\
\sum_{t=0}^{T-1}\left[\sum_{n \epsilon N_{t}} y_{n}\left(Z_{n} \cdot \theta_{n}\right)-\sum_{n \epsilon N_{t+1}} y_{n}\left(Z_{n} \cdot \theta_{a(n)}\right)\right]+\sum_{n \epsilon N_{T}} y_{n}\left(Z_{n} \cdot \theta_{n}\right)
\end{array}
$$

Observe that

$$
\sum_{n \in N_{t+1}} y_{n}\left(Z_{n} \cdot \theta_{a(n)}\right)=\sum_{n \in N_{t}}\left(\sum_{m \in C(n)} y_{m} Z_{m}\right) \cdot \theta_{n} .
$$

Hence,

$$
\begin{array}{r}
y_{0}\left(Z_{0} \cdot \theta_{0}\right)+\sum_{t=1}^{T} \sum_{n \epsilon N_{t}} y_{n}\left[Z_{n} \cdot\left(\theta_{n}-\theta_{a(n)}\right)\right]= \\
\sum_{t=0}^{T-1}\left[\sum_{n \epsilon N_{t}} y_{n}\left(Z_{n} \cdot \theta_{n}\right)-\sum_{n \epsilon N_{t}}\left(\sum_{m \epsilon C(n)} y_{m} Z_{m}\right) \cdot \theta_{n}\right]+\sum_{n \epsilon N_{T}} y_{n}\left(Z_{n} \cdot \theta_{n}\right)= \\
\sum_{t=0}^{T-1} \sum_{n \epsilon N_{t}}\left(y_{n} Z_{n}-\sum_{m \epsilon C(n)} y_{m} Z_{m}\right) \cdot \theta_{n}+\sum_{n \epsilon N_{T}} y_{n}\left(Z_{n} \cdot \theta_{n}\right)
\end{array}
$$

Q.E.D. 